\title{
2 The propofol binding sites of prokaryotic voltage-gated sodium channels
}

3 Elaine Yang ${ }^{1}$, Weiming $\mathrm{Bu}^{2}$, Antonio Suma ${ }^{3,6}$, Vincenzo Carnevale ${ }^{3}$, Kimberly C. Grasty ${ }^{4}$,

4 Patrick Loll ${ }^{4}$, Kellie Woll ${ }^{2}$, Natarajan Bhanu ${ }^{5}$, Benjamin A. Garcia ${ }^{5}$, Roderic G. Eckenhoff ${ }^{2}$ and Manuel

5 Covarrubias ${ }^{1,7}$

6

7

81 Department of Neuroscience, Sidney Kimmel Medical College, Thomas Jefferson University, 9 Philadelphia, PA

102 Department of Anesthesiology and Critical Care, Perelman School of Medicine, University of 11 Pennsylvania, Philadelphia, PA

$12{ }^{3}$ Institute for Computational Molecular Science, College of Science and Technology, Temple University, 13 Philadelphia, PA

144 Department of Biochemistry and Molecular Biology, College of Medicine, Drexel University, 15 Philadelphia, PA

$16{ }^{5}$ Department of Biochemistry and Biophysics, Perelman School of Medicine, University of Pennsylvania, 17 Philadelphia, PA

$18{ }^{6}$ Dipartimento di Fisica, Universit’a di Bari, and Sezione INFN di Bari, via Amendola 173, 70126

19 Bari, Italy

$20{ }^{7}$ Corresponding Author: Bluemle Life Sciences Building, 233 S $10^{\text {th }}$ Street, Room 231, Philadelphia, PA.

21 Manuel.Covarrubias@jefferson.edu. Tel. (215) 503-4341 


\section{Author Contributions}

24 EY - execution of electrophysiological and PAL experiments, analysis and interpretation of

25 electrophysiological results and creation of the corresponding figures; helped write and assemble the

26 manuscript.

$27 \mathrm{WB}$ - execution and analysis of PAL experiments, and creation of the corresponding figures; helped write

28 the corresponding methods section.

29 AS VC - creation of homology models; design, execution, analysis and interpretation of MD simulations, 30 and creation of associated figures; helped write corresponding methods, results and discussion.

31 PL KCG - overexpression and purification of Nav channel proteins, and creation of associated figures;

32 helped write the corresponding methods and results sections.

$33 \mathrm{KW}-$ execution and analysis of PAL experiments.

$34 \mathrm{NB}-$ execution and analysis of MS experiments

35 BG - provided state-of-the-art MS instrumentation and extended technical oversight and advice.

36 RGE - interpretation of the PAL results and their implications; helped coordinating the PAL work, and

37 edited of the manuscript.

$38 \mathrm{MC}$ - conceptual preparation of work, interpretation of results; coordination of all aspects of the work;

39 wrote manuscript and edited the final version.

40

41 


\section{Abstract}

43 Propofol, one of the most commonly used intravenous general anesthetics, modulates neuronal function by

44 interacting with ion channels. The mechanisms that link propofol binding to the modulation of distinct ion

45 channel states, however, are not understood. To tackle this problem, we investigated prokaryotic ancestors

46 of eukaryotic voltage-gated $\mathrm{Na}^{+}$channels (Navs) using unbiased photoaffinity labeling with a

47 photoacitivatable propofol analog $(\mathrm{AziP} m)$, electrophysiological methods and mutagenesis. The results

48 directly demonstrate conserved propofol binding sites involving the S4 voltage sensors and the S4-S5

49 linkers in $\mathrm{NaChBac}$ and NavMs, and also suggest state-dependent changes at these sites. Then, using

50 molecular dynamics simulations to elucidate the structural basis of propofol modulation, we show that the

51 S4 voltage sensors and the S4-S5 linkers shape two distinct propofol binding sites in a conformation-

52 dependent manner. These interactions help explain how propofol binding promotes activation-coupled

53 inactivation to inhibit Nav channel function. 
55 General anesthetics comprise a class of chemically heterogeneous drugs capable of simultaneously inducing 56 loss of consciousness, amnesia, immobilization, and analgesia through mechanisms that are not well

57 understood (Hemmings, 2019). Propofol, one of the most effective and widely used intravenous general

58 anesthetics, was invented in the late 1970's and is used for the induction and maintenance of sedation and

59 general anesthesia (Sahinovic, 2018; Walsh, 2018). Despite favorable properties, such as rapid induction

60 and recovery times, propofol also has significant negative cardiopulmonary side effects, including apnea,

61 hypotension, impaired myocardial contractility, and dystonia (Sahinovic, 2018). The hypnotic effects of

62 propofol result primarily from direct interactions with postsynaptic $\mathrm{GABA}_{\mathrm{A}}$ receptors, which potentiate

63 inhibitory GABA activity in the brain (Eckenhoff, 2018). However, general anesthesia likely involves

64 multiple targets that may be responsible for other endpoints and side effects (Eckenhoff, 2018; Hemmings,

65 2019). In particular, voltage-gated $\mathrm{Na}^{+}$channels (Navs), which are responsible for the initiation and

66 propagation of action potentials in the nervous system, may be one of these targets. Studies show that,

67 general anesthetics typically inhibit Navs (Ouyang, 2003, 2007; Barber, 2014; Sand, 2017; Yang, 2018). A

68 current hypothesis proposes that the negative modulation of Navs by general anesthetics may contribute to

69 the presynaptic effects of these drugs on neurotransmission (Herold, 2012; Zhou, 2019). This negative

70 modulation may result from various possible mechanisms in which anesthetic binding to Navs impairs ion

71 permeation and/or modifies gating. Determining the biophysical and structural bases of these mechanisms

72 is of paramount importance in the quest to understand the fundamental principles that explain how general

73 anesthetics affect the function of diverse ion channels and thereby induce physiological effects

74 (Covarrubias, 2015).

75 Recent work demonstrated that negative modulation of the prokaryotic Nav NaChBac by the inhalational

76 anesthetics isoflurane and sevoflurane may result from direct interactions that promote both activation and

77 inactivation (Ouyang, 2007; Barber, 2014; Kinde, 2016; Sand, 2017). We have also found that, in contrast

78 to the mechanism of local anesthetic action, propofol does not act as a pore blocker of $\mathrm{NaChBac}$ and another 
79 prokaryotic Nav NavMs (Yang, 2018). Instead, propofol renders voltage-dependent activation more

80 favorable, thereby promoting activation-coupled inactivation and, consequently, net negative modulation

81 (Yang, 2018). Furthermore, molecular dynamics simulations of the prokaryotic Navs in the

82 open/inactivated state revealed preferential propofol binding to an intersubunit hydrophobic pocket

83 involving the S4-S5 linker (Yang, 2018), which was corroborated by NMR-based experiments in NaChBac

84 (Wang, 2018). The S4-S5 linker plays a vital role in the electromechanical mechanisms of voltage-gated

85 ion channels by providing a physical connection between the voltage sensing and pore domains, (Catterall,

86 2010; Wisedchaisri, 2019). Therefore, propofol may act as a gating modifier of Navs that promotes

87 activation-coupled inactivation by interacting with the S4-S5 linker and neighboring regions. However, a

88 conclusive demonstration of propofol binding sites in Navs is still lacking. Understanding the structural

89 properties of these sites is a key step toward establishing the mechanism of propofol action.

90 To close this critical gap and render a structural model of propofol action, we performed unbiased

91 photoaffinity labeling (PAL) experiments using AziPm, a pharmacologically active diazirine derivative of

92 propofol (Hall, 2010). Results from NaChBac and NavMs show that propofol binds to critical pockets

93 involving the S4 voltage sensor and the S4-S5 linker. Moreover, a distinct photolabeling pattern of a non-

94 inactivating $\mathrm{NaChBac}$ mutant (T220A) suggests that these interactions are state-dependent. Then, using the

95 latest structural data from both prokaryotic and eukaryotic Navs, we conducted MD simulation studies to

96 produce a detailed atomistic model of how propofol modulates Navs by interacting with the

97 electromechanical apparatus in the resting, open, and inactivated states. The structural and mechanistic

98 insights provided by this study advance our understanding of how propofol modulates voltage-gated ion

99 channels, which may contribute to endpoints and side effects of propofol-induced anesthesia. 


\section{RESULTS}

\section{Modulation of NaChBac gating by AziPm}

103

To directly identify the propofol binding site in prokaryotic Navs, we employed an unbiased photoaffinity labeling (PAL) approach that has been extensively used to identify anesthetic binding sites of numerous proteins (Hall, 2010; Chiara, 2013; Weiser, 2013; Jayakar, 2014; Woll, 2016, 2017, 2018; Bensel, 2017). Here, we used AziPm (m-Azipropofol), an alkyl-diazirinyl derivative of propofol, to label propofol binding sites in NaChBac and NavMs (Hall, 2010). Though AziPm retains the pharmacological properties of propofol, we first sought to demonstrate that AziPm retains activity on prokaryotic Navs that is similar to that of the parent compound propofol.

Using whole-cell patch clamp electrophysiology, we characterized voltage-dependent gating of $\mathrm{NaChBac}$ heterologously expressed in HEK-293T cells, before and after exposure to 1 and $5 \mu \mathrm{M}$ AziPm (Figure 1A, Figure S1). Previously, we reported that propofol hyperpolarizes the voltage dependencies of both activation and inactivation, and accelerates macroscopic inactivation (Yang, 2018). Here, we found that the modulations of $\mathrm{NaChBac}$ by $\mathrm{AziP} m$ and propofol are very similar (Table 1). AziP $m$ induced parallel hyperpolarizing shifts of the peak conductance-voltage (G-V) relation (Figure 1B), without significant changes in the maximum conductance or effective gating charge of activation (Figure 2A, middle and right). At 1 and $5 \mu \mathrm{M}$ AziPm, the $\Delta \mathrm{V}_{1 / 2}$ of activation was $-11.29 \pm 1.44 \mathrm{mV}$ and $-12.34 \pm 1.19 \mathrm{mV}$, respectively (Figure 2A, left). We also characterized the time constants of current decay to evaluate macroscopic inactivation and found that AziP $m$ uniformly accelerates current decay between -40 and +60 $\mathrm{mV}$ in a concentration-dependent manner (Figure 1C). For example, the time constants of inactivation at $+40 \mathrm{mV}$ were $113 \pm 12$ and $54 \pm 6 \mathrm{~ms}$, in the presence of 1 and $5 \mu \mathrm{M}$ AziPm, respectively, compared to $158 \pm 12 \mathrm{~ms}$ for control (Table 1). AziP $m$ also induced parallel hyperpolarizing shifts of the pre-pulse inactivation curves (Figure 1D), without significant changes in the effective gating charge of inactivation (Figure 2B, right). The $\Delta \mathrm{V}_{1 / 2}$ of inactivation at 1 and $5 \mu \mathrm{M}$ were $-9.98 \pm 0.98 \mathrm{mV}$ and $-18.95 \pm 1.81 \mathrm{mV}$, 

in the channel availability at their respective baseline (control) $\mathrm{V}_{1 / 2}$ values of inactivation (Figure 2B,

\section{7 right)}

Overall, these results demonstrate that AziPm induces a relative stabilization of both the open and inactivated states and accelerates macroscopic inactivation (Table 1). These changes closely resemble the modulation of $\mathrm{NaChBac}$ and NavMs by propofol and can be explained by the previously proposed mechanism (Yang, 2018). Like propofol, AziPm primarily promotes voltage-dependent activation and consequently favors activation-coupled inactivation, which is ultimately responsible for the net inhibitory action at steady state. Therefore, the similar modulatory behaviors of both propofol and AziP $m$ reflects shared binding site(s) in prokaryotic Navs.

AziP $m$ binding to prokaryotic Nav channels

AziP $m$ contains a trifluorodiazirine substitution at the meta position that generates a highly reactive and non-selective carbene moiety upon UV photoactivation, allowing the unbiased identification of propofol binding sites in $\mathrm{NaChBac}$ and NavMs. Using affinity-purified detergent-solubilized NaChBac (Materials and Methods; Figure S2), we found AziPm photomodification at I124, S125 and N146 (Table 2; Figures S3a-d). Whereas adducts at I124 and S125 were detected in separate trials, the adduct at N146 was detected once (Table 2; Figures S3a-d). The adducted residues span a region that includes the end of the S4 voltage sensor (I124 and S125), the S4-S5 linker and the beginning of S5 in the pore domain (N146; Figures S6 and S7). This is a region that undergoes critical conformational changes during activation and inactivation 144 gating. Thus, to investigate a potential conformation dependence of propofol binding in $\mathrm{NaChBac}$ (Lee, 145 2012), we also performed photolabeling experiments on the non-inactivating NaChBac T220A mutant. 146 Previously, we reported that propofol induces robust positive modulation of this mutant by stabilizing the 147 open state in the absence of inactivation (Yang, 2018). We detected AziPm photomodification of NaChBac 

in WT NaChBac, were identified in separate attempts (Table 2; Figures S4a-b).

(Table 2; Figure S5a-c). I114 was also adducted in NavMs, a residue that is in close proximity to the equivalent NachBac residues, I124 and S125 (Figure S6). A sequence alignment that compares NaChBac and NavMs proteins demonstrates that the regional determinants of propofol binding are conserved in two distinct bacterial Nav channels. (Figure S7).

Consistent AziMPm photo-adduction limited to a discrete set of neighboring residues in two distinct bacterial Nav channels and a non-inactivating Nav mutant strongly suggests the presence of AziP $m$ binding pockets mainly involving the S4 segment and the S4-S5 linker in NaChBac and NavMs. Moreover, the

158 distinct photoadduction pattern of $\mathrm{NaChBac} \mathrm{T} 220 \mathrm{~A}$ suggests that propofol binding in $\mathrm{NaChBac}$ depends 159 on the gating conformation.

\section{MD simulations of Propofol binding to $\mathrm{NaChBac}$ in the inactivated state}

Under the conditions of the photoloabeling experiments, NaChBac is most likely in the inactivated state

162 (i.e., in the absence of a membrane potential). Thus, we characterized propofol binding to inactivated

$163 \mathrm{NaChBac}$ in silico by conducting molecular dynamics (MD) simulations in the presence of an excess of 164 propofol molecules to monitor binding near the experimentally observed sites, I124, S125, R131, and N146

165 (flooding MD simulations; Materials and Methods). Analysis of the four symmetry-related subunits of

166 NaChBac consistently demonstrate two specific binding cavities for propofol: one located near the C-

167 terminal end of S4 at the intracellular side of the voltage sensing domain, and the other, contiguous to the

168 first, at the N-terminal side of the S4-S5 linker (Figure 3A). Whereas R131 and S125 line the first pocket 169 and are in direct contact with bound propofol, N146 appears closer to the second pocket. I124 is in between 170 both pockets and its sidechain points toward the membrane (Figure 3B). To ascertain that photoadduction 171 events are possible between the bound propofol and sites I124, S125, R131, and N146, we calculated the 
172 minimum distance between these amino acids and the bound molecules of propofol for each configuration

173 sampled along the simulated trajectory (we considered both main- and side-chain groups). The bimodal

174 nature of the distribution of distances results from tumbling of the molecule within the binding pocket and

175 suggests loose hydrophobic interactions between propofol and the pocket. Consistent with the

176 photoadduction results, we found that propofol is within $4 \AA$ from S125, R131, N146 in a significant

177 fraction of the trajectory, which is $100 \mathrm{~ns}$ long (Figure 3C). By contrast, I124 appears to be farther away,

178 suggesting that its interaction with propofol occurs in a different conformation (i.e. resting or open). Also,

179 we observed no H-bonding between the 1-hydroxyl of propofol and groups in the channel protein, albeit a

180 water molecule appears to bridge the propofol's 1-hydroxyl to N146.

\section{Propofol binding to NaChBac models in the resting, activated/open and inactivated states}

182 To gain insights into the state-dependent character of the interaction between propofol and I124, S125,

183 R131 and N146, we used comparative homology modeling to generate structural models for the resting and

184 activated/open states (Materials and Methods). We first investigated whether or not the sidechains of the

185 photoadducted residues face the pockets in any of the conformational states. Then, a comparative analysis

186 of resting, activated/open, inactivated states focused on the size of the pockets allowed us to formulate

187 hypotheses on the binding of propofol to distinct conformational states.

188 The major change concerns S125 and R131, which line the voltage sensor domain pocket in the

189 activated/open and inactivated states, but not in the resting one (Figure 4). I124 is also particularly

190 interesting because its sidechain faces the membrane in the inactivated and open states and is in close

191 contact with N146 in the resting state, which may have a significant impact on photoadduction (Figure 4).

192 We then identified changes in the pocket geometry across the three states. The pocket facing the S4-S5

193 linker appears to be present only in the inactivated state (Figure 5). By measuring the number of waters

194 molecules within $6 \AA$ of residues L133 and I147 as a proxy for the pocket's volume, we confirmed that this

195 pocket is sufficiently large to bind propofol in the inactivated state only. By contrast, the site is collapsed 
in the resting and activated/open states (Figure 5). The pocket at the intracellular side of the voltage sensing

197 domain is present in both the activated/open and inactivated states, while showing a complete

198 reorganization in the resting one (as a result of the downward sliding of S4).

\section{Structural conservation of the propofol binding site in NaChBac and NavMs}

200

201

202

203

204

205

206

207

208

209

210

211

212

213

214

215

216

217

The electrophysiological and photoadduction experiments demonstrated extensive conservation in the ways in which propofol interacts with both $\mathrm{NaChBac}$ and NavMs (Table 2; Figures S6 and S7). To determine the structural correlates of this conservation, we compared the residues I124, S125, R131, N146 from NaChBac with the corresponding ones from the evolutionary related channel NavMs (PDB: 5HVX) (Sula et al., 2017). A structural superposition of the two structures in the open sate revealed that the equivalent NavMs residues are V111, S112, R118 and S133, respectively (Figure 6). This alignment confirms the importance of R118 (corresponding to R131 in $\mathrm{NaChBac}$ ) as a determinant of propofol binding in NavMs. I114 is not among the four residues, albeit it was also phototadducted in NaMs; however, the peptide bond atoms of this residue are in close proximity to other residues that may line the voltage sensor site (Figure 6).

\section{The hydroxyl group of propofol is not involved in NaChBac modulation}

The MD simulations of propofol binding to $\mathrm{NaChBac}$ and NavMs did not reveal critical H-bond interactions involving the 1-hydroxyl group and protein groups. To experimentally test the role of propofol's 1-hydroxyl group, we used fropofol, a propofol derivative in which the 1-hydroxyl group is replaced with an isosteric fluorine atom. Previously, we used fropofol to demonstrate that the propofol 1-hydroxyl group is necessary for the positive allosteric modulation of the ionotropic $\mathrm{GABA}_{\mathrm{A}}$ receptor and the induction of hypnosis in tadpoles (Woll, 2015). Thus, we proposed that hydrogen bonding involving the 1-hydroxyl group is necessary for propofol binding in $\mathrm{GABA}_{\mathrm{A}}$ receptors. 
218 We found that, in ways that are reminiscent of propofol action, fropofol also modulates activation and

219 inactivation gating (Figure 7A-C; Table 1). The time constants of current activation decreased gradually

220 at all voltages between -40 and $+40 \mathrm{mV}$ (Figure 7D). At $-40 \mathrm{mV}$, for example, the time constant of

221 activation was $24 \pm 3$ and $7 \pm 1 \mathrm{~ms}$ in the absence and presence of $5 \mu \mathrm{M}$ fropofol, respectively. Although

222 they do not exhibit significant voltage dependence between -40 and $+40 \mathrm{mV}$, fropofol decreased the time

223 constants of inactivation uniformly (Figure 7E). At +20 , for instance, the time constant of inactivation was

$224122 \pm 9 \mathrm{~ms}$ in the presence of $5 \mu \mathrm{M}$, compared to $168 \pm 10 \mathrm{~ms}$ for the control condition. Fropofol also

225 induced parallel hyperpolarizing shifts on the G-V relation and the pre-pulse inactivation curve. The $\Delta \mathrm{V}_{1 / 2}$

226 of activation and inactivation induced by fropofol was $-15.27 \pm 0.81 \mathrm{mV}$ and $-10.29 \pm 0.68 \mathrm{mV}$, respectively

\section{7 (Figure 7A-C; Table 1; Figure S9).}

228 These results demonstrate that the gating modulations of $\mathrm{NaChBac}$ by propofol and fropofol are overall

229 similar. Providing further support to this conclusion, fropofol recapitulates propofol action by inducing a

230 robust hyperpolarizing shift on the G-V relation of the non-inactivating mutant NaChBac T220A (Figure

231 S10). The fropofol-induced $\Delta \mathrm{V}_{1 / 2}$ of activation was $-14.72 \pm 1.28 \mathrm{mV}$ (Table 1). These results strongly

232 suggest that the 1-hydroxyl group of propofol is not involved in gating modulation of $\mathrm{NaChBac}$ by this

233 anesthetic. Together with the AziP $m$ photolabeling results, the electrophysiological characterizations of

234 AziPm and fropofol actions provide sound experimental constraints on the generation of structural models

235 to help explain propofol binding to prokaryotic Navs and the mechanism of gating modulation of these ion

236 channels by propofol. Furthermore, these results shed light on potential clinical implications of the

237 modulation of eukaryotic Navs by propofol. 


\section{DISCUSSION}

241 Previously, we demonstrated that propofol inhibits prokaryotic Navs by promoting activation-coupled

242 inactivation (Yang et a., 2018), and further computational work and NMR-based characterization suggested

243 that propofol mainly interacts with S4-S5 linker residues on the intracellular side of these Navs (Yang et

244 al., 2018, Wang et al., 2018). Thus, we hypothesized that propofol binds to pockets formed by the S4-S5

245 linker and neighboring regions, and that this interaction is responsible for the net negative allosteric

246 modulation of prokaryotic Navs. This is likely because the S4-S5 linker plays vital role in the gating

247 mechanisms of voltage-gated ion channels (Catterall, 2010). However, direct evidence for propofol binding

248 to the proposed pockets was lacking, and the possibility of gating-dependent conformational changes that

249 affect propofol binding to these pockets had not been examined. To tackle these problems, we investigated

250 NaChBac, NavMs, and a NaChBac mutant. Specifically, we used AziPm, a photoactivatable propofol

251 analog, to conduct unbiased photoaffinity labeling experiments coupled to electrophysiological analysis

252 and molecular dynamics simulations. The main results demonstrate that propofol binds to conserved

$253 \mathrm{NaChBac}$ and NavMs pockets formed by an intracellular region that spans the end of the S4 voltage sensor,

254 the S4-S5 linker and the beginning of the S5 pore segment. Furthermore, we provided biochemical and

255 computational evidence to suggest that the binding pockets undergo gating-dependent conformational

256 changes, and that the 1-hydroxyl group of propofol is not critical for propofol binding to prokaryotic Navs.

\section{The structural basis of the allosteric modulation of prokaryotic Navs by propofol}

258 Based on electrophysiological analysis and kinetic modeling that assumes preferential closed-state recovery

259 from inactivation, we previously suggested that propofol binding to $\mathrm{NaChBac}$ and NavMs accelerates the

260 rate constants of activation and inactivation without a change in the kinetics of recovery from inactivation

261 (Yang et al., 2018). Without a direct identification of the propofol binding site(s), however, the structural

262 correlates of this allosteric modulation remained speculative. The new photoadduction data and congruent

263 MD simulation results now provide physical evidence for two propofol binding pockets in a region that is

264 directly involved in the voltage-dependent communication between the voltage sensing domain and the 
pore domain. Propofol interacts with the intracellular side the S4 voltage sensor in one pocket, and the S4-

S5 linker in the other (Figs. 8-10). Suggesting a conformation-dependent interaction, the inactivating $\mathrm{NaChBac}$ wild type and the non-inactivating NaChBac T220A mutant yielded different photoadduction patterns. Whereas AziPm adducts were found at I124, S125 and N146 in the wild type, the adduct at R131 was only found in the non-inactivating mutant. R131 corresponds to R118 in NaMs, which was also photoadducted with AziPm (Table 2). This result was at first surprising because NavMs currents undergo

271 fast and complete inactivation. There are, however, important differences between these prokaryotic Navs

272 that must be considered to understand this result. Compared to NaChBac, NavMs exhibits faster activation

273 and inactivation kinetics and operates over a voltage range that is $30-40 \mathrm{mV}$ more hyperpolarized (Yang

274 et al., 2018). Furthermore, their S4 and S4-S5 linkers are only 35\% identical, and the photoadducts may

275 form at the peptide bonds rather than the amino acid sidechains. Given these considerations, subtle

276 structural differences in the propofol pockets between NavMs and NaChBac may explain why equivalent

277 photoadducts are observed in potentially distinct conformations. Consistent with this possibility, a second

278 photoadduct was found at I114 in NavMs, a position that is only two residues downstream from a position

279 equivalent to S125 in NaChBac. Although we observed no exact match between the AziPm photoadducts

280 detected in NaChBac and NavMs, it is striking that the vast majority of them were found in the S4-S5 linker

281 or in close proximity to it in 8/9 reactions (Table 2). We therefore conclude that the S4-S5 linker and

282 neighboring regions are direct physical determinants of propofol binding in prokaryotic Navs. This

283 conclusion is also supported by the results of a previous study that used ${ }^{19} \mathrm{~F}-\mathrm{NMR}$ saturation transfer

284 difference spectroscopy to investigate the binding of 4-fluoropropofol to potential sites suggested by

285 docking in silico (Wang et al., 2018). This study, however, identified additional sites in the pore, the

286 external apex of the voltage sensor and the internal activation gate, which were not observed in the PAL

287 experiments reported here. Various factors could have contributed to this discrepancy. For instance, NMR

288 experiments required cysteine mutations at predetermined putative sites to label the protein with the NMR

289 probe. These mutations and the probe can have effects on gating and propofol binding. These experiments

290 also required concentrations of the fluorinated ligand that are $40-200$ times higher than the concentrations 
of AziP $m$ used in PAL experiments and, therefore, the additional sites might be low affinity sites that were not detected in our experiments. results and MD simulations extended to major gating states, helps now formulate a structure-based model to explain the mechanism of the allosteric modulation of prokaryotic Navs by propofol. Primarily, binding movement of the S4 segment and thereby promote complete activation and pore opening. In turn, this positive modulation, could also indirectly favor inactivation of open channels, which may start in the selectivity filter and propagate to the rest of the pore domain (Pavlov et al., 2005; Gamal El-Din et al., 2019). Propofol may also have catalytic allosteric effects on inactivation gating through its binding to the S4-S5 linker pocket in a putative inactivated state. This interaction is especially intriguing because this pocket in $\mathrm{NaChBac}$ and $\mathrm{NavMs}$ corresponds to a hydrophobic cavity recently identified as the binding site of the IFM motif in the eukaryotic Nav1.4 and Nav1.5 (Pan et al., 2018; Jiang et al., 2020). This motif is the "inactivation particle" of most eukaryotic Nav channels, which acts allosterically on the pore domain to induce fast inactivation (Catterall, 2012; Ahern et al., 2015).

\section{Conservation of general anesthetic sites involving the S4-S5 linker of diverse ion channels}

307 Using photoaffinity labeling, previous work from our laboratories have provided strong evidence to suggest that the S4-S5 linker and neighboring regions of Kv and TRPA1 channels are critical structural determinants of their modulation by volatile (sevoflurane) and intravenous (propofol) anesthetics (Bu et al., 2017; Woll et al., 2017). Based on the new results reported here, we can reach a similar conclusion for prokaryotic

311 Navs. There is, however, little to no identity between the S4-S5 linkers of distantly related Kv, TRPA1 and

312 Nav channels, albeit they all have similar topological features (6 transmembrane, 6TM, segments) and

313 overall architecture. Therefore, we propose that common physical-chemical properties (e.g., amphipathic 314 character) and a similar shape of the cavities formed by this linker and neighboring regions (the S6 segment 

binding determinants of general anesthetics and are directly involved in the mechanism of action.

\section{Mechanistic implications for eukaryotic Navs}

Whether or not the hydrophobic IFM cavity also binds propofol in eukaryotic Navs needs to be determined.

Previous studies have demonstrated that propofol and volatile anesthetics inhibit mammalian Navs, and have suggested that this inhibition may depress excitatory synaptic transmission in the brain, but the molecular mechanism of this inhibition is not known (Ratnakumari and Hemmings, 1997; Lingamaneni,

323 Birch and Hemmings, 2001; Ouyang, Wang and Hemmings, 2003; Herold and Hemmings, 2012). The

324 study of prokaryotic Navs with a simpler homotetrameric structure has helped us generate a sound working

325 hypothesis that explains how propofol can modulate Nav channel activation and inactivation gating through

326 allosteric interactions that involve the S4 voltage sensor and the S4-S5 linker. Although these regions have

327 structural features that are generally conserved in all Navs, eukaryotic Navs are more complex pseudotetrameric proteins made of four homologous domains; therefore, characterizing the mechanism of propofol action and its structural basis in detail has been intrinsically difficult (Catterall, 2012). Recent advances, however, have made possible the structural characterization of eukaryotic Navs with atomic resolution,

331 providing avenues to visualize their interactions with toxins, anesthetics and other drugs (Pan et al., 2018;

332 Shen et al., 2019; Jiang et al., 2020).

Nav channel modulation by propofol and the end points of general anesthesia

334 Negative modulation of Navs would inhibit excitation of neurons and muscles and could be linked to common endpoints of general anesthesia, such as loss of consciousness, amnesia, analgesia and

336 immobilization. Most likely, however, propofol-induced sedation and loss of consciousness result from 337 potentiation of GABA receptors (Tang and Eckenhoff, 2019). In support of this conclusion, the propofol 338 derivative fropofol, which has the 1-hydroxyl group replaced with an isosteric fluorine, does not interact 
339 with the $\mathrm{GABA}_{\mathrm{A}}$ receptor and has no hypnotic activity (Woll et al., 2018). It was thus proposed that the 1-

340 hydroxyl group of propofol participates in hydrogen bonding that is critical for the hypnotic activity of

341 propofol resulting from its interaction with $\mathrm{GABA}_{\mathrm{A}}$ receptors. In contrast, we found that propofol and

342 fropofol have a very similar profile of activity on the prokaryotic NaChBac, indicating that H-bonding

343 mediated by the propofol's hydroxyl group does not play a critical role in a prokaryotic Nav (Figure 6).

344 We do not know whether this would also be the case in eukaryotic Navs; however, such a difference would

345 suggest that the documented inhibition of mammalian Navs by propofol may rather be linked to other

346 pharmacological properties (e.g., muscle relaxant and anti-epileptic) or toxicity associated with myocardial

347 depression (Lundstrom et al., 2010; Woll et al., 2015).

\section{Conclusions}

349 We used a set of complementary approaches including electrophysiological analysis, mutagenesis, unbiased

350 PAL, MD simulations and a manipulation of propofol chemistry to identify the propofol binding sites in

351 two prokaryotic Navs and determine a plausible mechanism of action. We conclude that propofol binds to

352 two contiguous binding cavities. One involving the cytoplasmic side of the S4 voltage sensor and the other

353 involving the S4-S5 linker and part of the S5 segment. State-dependent interactions with these sites are the

354 basis of a mechanism in which propofol promotes activation-coupled inactivation to induce net inhibition

355 of the Navs. Given that the interactions do not depend on the 1-hydorxyl group of propofol, we hypothesize

356 that the modulation of eukaryotic Navs by propofol may not be involved in the hypnotic effects of this

357 anesthetic. 


\section{MATERIALS AND METHODS}

Molecular and Cell Biology

Wild-type (WT) NaChBac cDNA in a modified pTracer-CMV2 expression vector was a gift from D. Ren

(University of Pennsylvania, Philadelphia, PA). cDNA was amplified in bacterial culture and purified with

plasmid using the QuikChange site-directed mutagenesis method (Agilent). HEK-293 cells were transiently

transfected with $\mathrm{NaChBac}$ WT or T220A cDNA using Lipofectamine ${ }^{\mathrm{TM}} 2000$ transfection reagent

(Invitrogen) and seeded onto $12 \mathrm{~mm}$ circular glass coverslips 24 hours prior to patch clamp recording.

Standard protocols were followed for growth and maintenance of cells in culture.

367

368

369

370

371

372

373

374

375

376

377

378

379

380

\section{Electrophysiology}

Patch pipettes were pulled from borosilicate capillary glass (LA16, Dagan Corp.) with a HEKA PIP6 micropipette puller (HEKA). Prior to recording, patch pipettes were fire polished to a final resistance of 1.5 to 2.3 M $\Omega$. Whole-cell patch clamp recording was performed using an Axopatch 200B amplifier (Molecular Devices) and Digidata 1440A analog-to-digital converter (Molecular Devices). Series resistance was compensated at least $85 \%$. Passive leak current and capacitive transients were subtracted online by standard $\mathrm{P} / 4$ protocol. All recordings were low-pass Bessel-filtered at $2 \mathrm{kHz}$ and digitized at $15.4 \mathrm{kHz}$. Clampex 10 (pCLAMP 10, Molecular Devices) was used to control voltage protocols and for data acquisition.

The extracellular bath solution contained (in $\mathrm{mM}$ ) $140 \mathrm{NaCl}, 4 \mathrm{KCl}, 1.5 \mathrm{CaCl} 2,1.5 \mathrm{MgCl} 2,10 \mathrm{HEPES}$, and 5 D-glucose, $\mathrm{pH} 7.3$ adjusted with $\mathrm{NaOH}$; the intracellular pipette solution contained (in $\mathrm{mM}$ ) $15 \mathrm{NaCl}$, $80 \mathrm{CsF}, 40 \mathrm{CsCl}, 10 \mathrm{EGTA}$, and $10 \mathrm{HEPES}, \mathrm{pH} 7.3$ adjusted with $\mathrm{CsOH}$. AziPm was synthesized by previously published methods (Hall, 2010). Prior to use, AziPm was diluted in bath solution to working concentrations, followed by alternating sonication and vortexing for 4.5 minutes. All dilutions were prepared and utilized the same day. Cells were continuously perfused with bath solution at room 
381 temperature $\left(22-25^{\circ} \mathrm{C}\right)$ during recordings. In all experiments, all control recordings were collected first,

382 prior to any AziPm exposure. Following control recordings, AziP $m$ was perfused for $\sim 3$ minutes before

383 collecting paired anesthetic recordings and continuously thereafter. To prevent inaccuracies due to any

384 membrane lipid retention of anesthetic molecules and cumulative effects, each experimental cell was only

385 exposed to a single concentration of AziPm, and washout data was not used.

\section{Voltage Protocols}

In NaChBac WT and T220A, voltage-dependent activation was assessed with $\mathrm{Na}+$ currents evoked by 500$\mathrm{ms}$ depolarizing steps $(-100$ to $+60 \mathrm{mV}, \Delta \mathrm{V}=10 \mathrm{mV})$ from a holding potential of $-120 \mathrm{mV}$. Pre-pulse inactivation was assessed with a two-pulse protocol: (1) a 2 -s conditioning pulse (-120 $\mathrm{mV}$ to $-10 \mathrm{mV}, \Delta \mathrm{V}$ $=10 \mathrm{mV}$ ), followed immediately by (2) a 50-ms test pulse to $+10 \mathrm{mV}$. The holding potential was $-120 \mathrm{mV}$.

Analysis of Electrophysiological Results

Clampfit 10 (pCLAMP 10, Molecular Devices), Origin 9.1 (OriginLab Corp.) and Excel 2013 (Microsoft

393 Corp.) were used to analyze voltage-clamp data. All evaluated parameters are reported as mean \pm SEM.

394 The paired samples t-test was used to assess differences between paired data sets in the absence and 395 presence of propofol. P-values less than 0.05 are explicitly reported in the figures and figure legends, and 396 "N.S." indicates $\mathrm{P} \geq 0.05$.

397 Peak chord conductance (G) was calculated using $G=I /\left[V-V_{\text {rev }}\right]$, where $I$ is the measured peak current, $398 V$ is the command potential, and $V_{\text {rev }}$ is the reversal potential extrapolated from individual current-voltage 399 curves. The voltage dependence of activation ( $\mathrm{G}-\mathrm{V}$ curve) was derived from the best-fit fourth order 400 Boltzmann function $G(V)=\left[G_{\max } /\left(1+e^{(V s-V) / k}\right)\right]^{4}$ and normalized to $G_{\max }$, where $G_{\max }$ is the maximum 401 peak conductance, $V_{s}$ is the midpoint of activation for a single subunit, $V$ is the command potential and $k$ is 402 the slope factor. The midpoint voltage of activation was calculated using $V_{1 / 2}=\left(V_{s}+1.67 \mathrm{k}\right)$. Pre-pulse inactivation parameters were determined from the best-fit first order Boltzmann function $I(V)=I_{\max } /(1+$ 
$\left.404 e^{(V I / 2-V) / k}\right)$ and normalized to $I_{\max }$, where $I_{\max }$ is the maximum current amplitude and $V_{1 / 2}$ is the midpoint 405 voltage of inactivation. Time constants of activation and inactivation were derived from the rising and 406 decaying components of the $\mathrm{Na}^{+}$current, respectively, using the best-fit single exponential of the form $I(t)$ $407=\left(A e^{-t / \tau}+C\right)$, where $A$ is the amplitude, $C$ is the plateau constant, $t$ is time, and $\tau$ is the time constant of 408 activation or inactivation.

410 The structural model of the open state was obtained using comparative homology modeling based on the 411 cryoEM structure of the eukaryotic voltage-gated sodium selective channel NavPas (PDB: 6a90; Shen, et 412 al., 2018). The inactivated conformation was modelled using NavAb as template (PDB: 5vb2; Lenaeus, et 413 al., 2017). For the resting state we used the recently published xray structures of NavAb in the resting state 414 (PDB: 6p6w; Wisedchaisri, et al., 2019). We threaded the sequence of NaChBac through the three PDB 415 structures using the SWISS-MODEL software [Waterhouse, et al., 2018). The accuracy of the NaChBac 416 model in the inactivated state was confirmed by comparing it against the recently published cryoEM 417 structure of potentially inactivated NaChBac (Figure S8) (Gao et al., 2020).

419 Molecular systems were assembled using the CHARMM-GUI webserver (Wu, et al., 2014). Each channel 420 state was embedded in a lipid bilayer of POPC, and the number of ions in the bulk was set to $0.15 \mathrm{M} \mathrm{KCl}$ 421 and to obtain electrical neutrality. The molecular systems, included waters, contains about 180000 atoms. 422 Molecular dynamics calculations are performed using the NAMD computational code (Phillips et al., et al., 423 2005), using the all-atom potential energy function CHARMM36 for protein and phospholipids, and the 424 TIP3P potential for water molecules (Mackerell, et al., 2004; Jorgensen, et al., 1983). Periodic boundaries 425 conditions are applied, and long-range electrostatic interactions are treated by the particle mesh Ewald 426 algorithm (Essmann, et al., 1995). The Propofol molecule was modeled using the CGenFF webserver 427 (Vanommeslaeghe, et al., 2012a; Vanommeslaeghe, et al., 2012b). Molecular systems are equilibrated for 428 about $1 \mathrm{~ns}$ with decreasing harmonic restraints applied to the protein atoms and the lipid membrane. All 
429 trajectories are generated with a time step of $2 \mathrm{fs}$ at constant normal pressure $(1 \mathrm{~atm})$ controlled by a

430 Langevin piston and constant temperature (300 K) using a Nosé-Hoover thermostat. The configurations

431 and results presented in Fig. 4D and Fig. 5 were obtained after simulating the three systems for about 100ns.

432 Measurements related to Fig. 3A-C were obtained using flooding simulations based on the same protocol

433 used previously (Wang, et al., 2018).

\section{Expression and Purification of NavMs and NaChBac}

435 The genes for NaChBac and NavMs were amplified using appropriate PCR primers (Table S1) and 436 subcloned into the in-house plasmid pETHT. This vector encodes an N-terminal His 6 tag followed by a

437 TEV protease recognition sequence, which is followed immediately by the sequence of the protein to be

438 expressed. Both genes were inserted between the BsaI and NotI sites of the vector. The T220A mutant of

439 NaChBac was prepared using PCR-mediated site-directed mutagenesis, with the primers shown in Table 440 S1.

441 The NavMs plasmid was transformed into E. coli C41 (DE3). A single colony was diluted in LB medium 442 containing $100 \mathrm{ug} / \mathrm{mL}$ ampicillin and grown overnight. The following morning, $20 \mathrm{~mL}$ of overnight culture 443 were diluted into $1 \mathrm{~L}$ of $\mathrm{LB}+$ ampicillin, and the flasks were shaken at $37^{\circ} \mathrm{C}$ until $\mathrm{OD}_{600}$ of $0.6-0.8$, at which 444 point IPTG was added at a final concentration of $0.5 \mathrm{mM}$. Cells were harvested $3.5 \mathrm{~h}$ after induction and 445 frozen at $-80^{\circ}$ until use.

446 All purification steps were carried out at $4{ }^{\circ} \mathrm{C}$. Cell pellets were thawed, re-suspended in lysis buffer $(20$

$447 \mathrm{mM}$ Tris-Cl, $\mathrm{pH}$ 7.5, $300 \mathrm{mM} \mathrm{NaCl}, 10 \mathrm{mM}$ imidazole), and lysed by three passes through an ice-cold 448 Avestin Emusiflex C5 cell disrupter. Cell lysates were clarified by centrifugation at 15,000xg for 15 mins; 449 the cleared lysate was then centrifuged at 145,000xg for $1 \mathrm{hr}$. The resulting pellet was homogenized in lysis 450 buffer, after which dodecyl maltoside was added to a final concentration of $1 \%(\mathrm{w} / \mathrm{v})$. The solution stirred 451 gently at $4{ }^{\circ} \mathrm{C}$ for $1 \mathrm{hr}$ then centrifuged at $145,000 \mathrm{x} g$ for $1 \mathrm{hr}$. The supernatant was filtered using a Millex452 SV 5.0 um syringe filter (Merck-Millipore) and loaded onto a 1-mL HiTrap IMAC-HP column (GE 
453 Healthcare), which was pre-equilibrated with lysis buffer containing $0.1 \%$ dodecyl maltoside. The column

454 was washed and then eluted with $50 \mathrm{mM}$ Tris-Cl, $\mathrm{pH}$ 7.7, $500 \mathrm{mM} \mathrm{NaCl}, 500 \mathrm{mM}$ imidazole, $0.1 \%$ dodecyl

455 maltoside. The purified protein was then dialyzed against $50 \mathrm{mM}$ Tris, $\mathrm{pH}$ 7.7, $500 \mathrm{mM} \mathrm{NaCl,} \mathrm{0.1 \%} \mathrm{DDM,}$

456 and concentrated to a final concentration of 2-5 mg/mL (Figure S2).

$457 \mathrm{NaChBac}$ was expressed and purified in the same manner as NavMs, with the following modifications.

458 After dilution of the original overnight culture, the flasks were shaken at $30{ }^{\circ} \mathrm{C}$ until an $\mathrm{OD}_{600}$ of $0.6-0.8$

459 was reached, at which point IPTG was added and the temperature was reduced to $18{ }^{\circ} \mathrm{C}$; cells were

460 harvested $24 \mathrm{~h}$ after induction; cell pellets were disrupted in a lysis buffer containing $50 \mathrm{mM}$ Tris, $\mathrm{pH}$ 7.7,

$461500 \mathrm{mM} \mathrm{NaCl}, 0.2 \mathrm{mM}$ PMSF, $2 \mathrm{mg} / \mathrm{mL}$ lysozyme, $2 \mu \mathrm{g} / \mathrm{mL}$ DNase; and the detergent solubilization step

462 was carried out overnight (Figure S2).

Photolabeling of NaChBac WT, NaChBac T220A, and NavMs

464 A final concentration of $5 \mu \mathrm{M}$ AziP $m$ with or without $200 \mu \mathrm{M}$ propofol was added to the purified $\mathrm{NaChBac}$,

$465 \mathrm{NaChBac}$ T220A, and NavMs to a final protein concentration of $1 \mu \mathrm{g} / \mu \mathrm{l}$. The samples were equilibrated on

466 ice in the dark for $5 \mathrm{~min}$ and then irradiated for $25 \mathrm{~min}$ at $350 \mathrm{~nm}$ with a RPR-3000 Rayonet lamp in 1-mm

467 path length quartz cuvettes through a 295-nm glass filter (Newport Corporation).

In-Solution Protein Digestion

469 After UV exposure proteins were precipitated overnight at $-20^{\circ} \mathrm{C}$ in 4 volumes of chilled acetone. Protein 470 was pelleted for $20 \mathrm{~min}$ at $16,000 \mathrm{xg}$ at $4{ }^{\circ} \mathrm{C}$ then gently washed twice with $300 \mu$ l of chilled acetone.

471 Protein pellets were air-dried before resuspension in $50 \mu \mathrm{l}$ of $50 \mathrm{mM}$ Tris- $\mathrm{HCl}, \mathrm{pH} 8.0,1 \%$ Triton X-100,

472 and $0.5 \%$ SDS. Insoluble debris was pelleted by centrifugation at $16,000 \mathrm{x}$. The samples were 473 resuspension in final concentration of $50 \mathrm{mM} \mathrm{NH}_{4} \mathrm{HCO}_{3}$. Following $1 \mu \mathrm{L} 0.5 \mathrm{M}$ dithiothreitol (DTT) was 474 added and samples were incubated at $56^{\circ} \mathrm{C}$ for $30 \mathrm{~min} .0 .55 \mathrm{M}$ iodoacetamide (IAA) was then added and 475 protein samples were incubated at room temperature in the dark for $45 \mathrm{~min}$. Sequencing grade-modified 
476 trypsin (Promega) was added to a final 1:20 protease: protein ratio (w:w) with additional of $0.2 \%(\mathrm{w} / \mathrm{v} \%)$

477 ProteaseMax TM Surfactant. Proteins were digested overnight at $37^{\circ} \mathrm{C}$. Trypsin digested peptides were 478 diluted to $200 \mu \mathrm{L}$ with final concentration of $100 \mathrm{mM} \mathrm{NH}_{4} \mathrm{HCO}_{3}$ and $0.02 \%$ ProteaseMAX Surfactant prior 479 to the addition of sequencing grade chymotrypsin (Promega) to a final 1:20 protease:protein ratio (w:w).

480 Proteins were digested overnight at $37^{\circ} \mathrm{C}$. Acetic acid $(\mathrm{AcOH})$ was added to until the $\mathrm{pH}<2$ and the peptide

481 digests were incubated at room temperature for $10 \mathrm{~min}$ prior to centrifugation at $16,000 \mathrm{xg}$ for $20 \mathrm{~min}$ to

482 remove insoluble debris. The sample was desalted using C18 stage tips prepared in house. Samples were

483 dried by speedvac and resuspended in $0.1 \%$ formic acid immediately prior to mass spectrometry analysis.

\section{In-Gel Protein Digestion}

Photolabeled proteins were separated by SDS-PAGE. The identified bands corresponding to NaChBac and NavMs were excised. Excised bands were distained, dehydrated and dried by speed vac before proteins were reduced by incubation at $56^{\circ} \mathrm{C}$ for $30 \mathrm{~min}$ in $5 \mathrm{mM}$ DTT and $50 \mathrm{mM} \mathrm{NH} 4 \mathrm{HCO}_{3}$. The DTT solution was removed and proteins were then alkylated by the addition of $55 \mathrm{mM} \mathrm{IAA}$ in $50 \mathrm{mM} \mathrm{NH} \mathrm{NCO}_{3}$ and incubation at room temperature for $45 \mathrm{~min}$ in the dark. Bands were dehydrated and dried by speed vac before resuspension in $100 \mu \mathrm{L} 0.2 \%$ ProteaseMAX ${ }^{\mathrm{TM}}$ surfactant(Promega) and $50 \mathrm{mM} \mathrm{NH}_{4} \mathrm{HCO}_{3}$ solution containing trypsin (sequencing grade, Promega) at a 1:20 protease:protein ratio (w:w). Proteins were 492 digested for $12-16 \mathrm{hrs}$ at $37^{\circ} \mathrm{C}$. After trypsin digestion, the samples were diluted to $200 \mu \mathrm{L}$ with final 493 concentration of $100 \mathrm{mM} \mathrm{NH}_{4} \mathrm{HCO}_{3}$ and $0.02 \%$ ProteaseMAX 494 digested overnight at $37{ }^{\circ} \mathrm{C}$ with the addition of sequencing grade chymotrypsin (Promega) to a final 495 protease:protein (w:w) ratio of 1:20. Multiple peptide extractions were performed in order to increase 496 hydrophobic peptide retrieval from the gel. The peptide extractions were pooled from the different ratio of 497 acetylnitrile and acetic acid. The final extractions were sonicated for $20 \mathrm{~min}$ and dried by speed vac before resuspension in $0.5 \%$ acetic acid and further acidified until the $\mathrm{pH}<2$. Samples were sonicated for $10 \mathrm{~min}$ 
500 C18 stage tips prepared in house. Samples were dried by speed-vac and resuspended in $0.1 \%$ formic acid

501 immediately prior to mass spectrometry analysis.

\section{Mass Spectrometry}

503 Mass analysis was performed similar to as previously reported (Hall, 2010; Woll, 2017). Briefly, desalted 504 peptides were injected into a Thermo LTQ Orbitrap XL Mass Spectrometer (Thermo Fisher Scientific, 505 Waltham, MA, USA) or an Orbitrap Elite ${ }^{\mathrm{TM}}$ Hybrid Ion Trap mass spectrometer. Peptides were eluted with 506100 min with linear gradients of $\mathrm{ACN}$ in $0.1 \%$ formic acid in water (v/v\%) starting from $2 \%$ to $40 \%(85$ $507 \mathrm{~min})$, then $40 \%$ to $85 \%$ (5 $\mathrm{min})$ and finally $85 \%$ (10 $\mathrm{min})$.

508 Spectral analysis was conducted using Maxquant (Thermo Scientific) to search b and y ions against the 509 sequence containing NaChBac and NavMs. All analyses included dynamic oxidation of methionine $510(+15.9949 \mathrm{~m} / \mathrm{z})$ as well as static alkylation of cysteine $(+57.0215 \mathrm{~m} / \mathrm{z}$; iodoacetamide alkylation). Filter 511 parameters were Xcorr scores $(+1$ ion) 1.5 , ( +2 ion) $2.0,(+3$ ion) 2.5 , deltaCn 0.08 , and peptide probability

512 0.05. Photolabeled peptides were searched with the additional dynamic AziPm modifications. Both the in513 solution and in-gel sequential trypsin/chymotrypsin digests were searched without enzyme specification 514 with a false discovery rate of 0.01 . Samples were conducted in triplicate and samples containing no 515 photoaffinity ligand were treated similarly to control for false positive detection of photoaffinity ligand 516 modifications. To confirm the photolabeled adduct, MS work was repeated at the Proteomics Core Facility 517 of the Wistar Institute (University of Pennsylvania, Philadelphia, PA, USA). 


\section{ACKNOWLEDGMENT}

520 This work was supported by following grants from the National Institutes of Health, USA: P01GM55876

521 (MC, VC, RGE), 1R01NS111997-01A1 (BAG), F30GM123612 (EY). We also thank members of the

522 Covarrubias lab for their constructive feedback and support. 
Figure 1. Modulation of NaChBac by AziPm. A) Families of whole-cell inward currents evoked by increasing step depolarizations from a holding voltage of $-120 \mathrm{mV}(-100$ to $+60 \mathrm{mV}, \Delta \mathrm{V}=10 \mathrm{mV})$. The start-to-start interval was $10 \mathrm{~s}$. The inset shows scaled currents at $0 \mathrm{mV}$. B) Normalized peak conductancevoltage relations. The solid lines are best-fit $4^{\text {th }}$ order Boltzmann functions (summary of best-fit parameters on Table 1). C) Time constants of current decay against step depolarization voltage. The time constants were derived from fitting an exponential function to the decay of the current (Materials and Methods). D) Normalized pre-pulse inactivation curves. The currents were evoked by a constant step depolarization from increasing conditioning depolarizations as indicated on the graph The solid lines are best-fit $1^{\text {st }}$ order Boltzmann functions (summary of best-fit parameters on Table 1). NaChBac was expressed in HEK293T cells (Materials and Methods). Source Data 1-5.

Figure 2. Statistical analysis of changes in NachBac gating parameters induced by 1 and $5 \mathrm{mM}$

AziPm. A) and B) Scatter graphs of $\mathrm{V}_{1 / 2}, \mathrm{G}_{\max }$ and $\mathrm{z}$ derived from the best-fit to $4^{\text {th }}$-order Boltzmann functions (Materials and Methods). C) and D) Scatter graphs of $V_{1 / 2}, I_{\max }$ and $z$ derived from the best-fit to $1^{\text {st }}$ order Boltzmann functions (Materials and Methods). The paired Student's t-test was used to evaluate the changes. The $\mathrm{p}$ values are indicated in each graph (N.S. = not significant). Source Data 1-5.

Figure 3. Binding of propofol to NaChBac in the inactivated state. A) Intracellular view of the tetrameric assembly. The voltage sensing (S1-S4) and pore domains (S5, S6) are colored in light blue and molecules are shown occupying two distinct pockets. One at the end of the S4 helix, and the other in close contact with the S4-S5 linker. B) Close up of the region highlighted in A), with amino acids I124 (orange), S125 (red), R131 (green) and N146 (blue) propofol (grey) shown as sticks. C) Minimum distance distribution between I124, S125, R131, N146 and propofol molecules. 
547 Figure 4. Models of the resting, open, inactivated states of NaChBac. Close ups of the propofol binding

548 sites as described in Figure 3 legend. The voltage sensing (S1-S4) and pore domains (S5, S6) are colored

549 in light blue and purple, respectively, and the S4-S5 linker connecting these domains is colored in yellow.

550 The interacting amino acids are highlighted.

551 Figure 5. NaChBac models of resting, open, inactivated states. Molecular surfaces and colored 552 according to the distinct symmetry related subunits. The S4-S5 linker pocket is highlighted in the 553 inactivated state. Histograms show the number of water molecules that are closer than $6 \AA ̊$ from amino acids $554 \quad$ L133 and I147.

555 Figure 6. Structural superposition between NavMs and NaChBac. The amino acids aligned to I124, 556 S125, R131, N146 are V111, S112, R118 and S133. I114 is also highlighted in violet.

557 Figure 7. Modulation of NaChBac by fropofol. A) Families of whole-cell inward currents evoked by 558 increasing step depolarizations from a holding voltage of $-120 \mathrm{mV}(-100$ to $+60 \mathrm{mV}, \Delta \mathrm{V}=10 \mathrm{mV})$. The 559 start-to-start interval was $10 \mathrm{~s}$. The overlay shows scaled currents at $0 \mathrm{mV}$. B) Normalized peak 560 conductance-voltage relations. The solid lines are best-fit $4^{\text {th }}$ order Boltzmann functions (summary of best-

561 fit parameters on Table 1). C) Normalized pre-pulse inactivation curves. The currents were evoked by a 562 constant step depolarization from increasing conditioning depolarizations as indicated on the graph 563 (Materials and Methods). The solid lines are best-fit Boltzmann functions (summary of best-fit parameters 564 on Table 1). D) Time constants of current activation against step depolarization voltage. The time constants 565 were derived from fitting an exponential function to the rising phase of the current (Materials and Methods). 566 E) Time constants of current decay (inactivation) against step depolarization voltage. The time constants 567 were derived from fitting an exponential function to the decay of the current (Materials and Methods). $568 \mathrm{NaChBac}$ was expressed in HEK293T cells (Materials and Methods). Source data 6-8. 


\section{REFERENCES}

570

571

572

573

574

575

576

577

578

579

580

581

582

583

584

585

586

587

588

589

590

591

592

593

594

595

Ahern, C. A., Payandeh, J., Bosmans, F. \& Chanda, B. (2015). The hitchhiker's guide to the voltagegated sodium channel galaxy. J. Gen. Physiol. 147, 1-24. doi: 10.1085/jgp.201511492.

Barber, A. F., Carnevale, V., Klein, M. L., Eckenhoff, R. G., \& Covarrubias, M. (2014). Modulation of a voltage-gated $\mathrm{Na}+$ channel by sevoflurane involves multiple sites and distinct mechanisms. Proc. Natl. Acad. Sci. U. S. A., 111(18), 6726-6731. doi: 10.1073/pnas.1405768111.

Bu, W., Liang, Q., Zhi, L., Maciunas, L., Loll, P. J., Eckenhoff, R.G. \& Covarrubias, M. (2018). Sites and Functional Consequence of Alkylphenol Anesthetic Binding to Kv1.2 Channels. Mol. Neurobiol. 55(2):1692-1702. doi: 10.1007/s12035-017-0437-2.

Bensel, B. M., Guzik-Lendrum, S., Masucci, E. M., Woll, K. A., Eckenhoff, R. G., \& Gilbert, S. P. (2017). Common general anesthetic propofol impairs kinesin processivity. Proc. Natl. Acad. Sci. U. S. A., 114(21), E4281-E4287. doi: 10.1073/pnas.1701482114.

Catterall, W. A. (2010). Ion channel voltage sensors: Structure, function, and pathophysiology. Neuron. doi: 10.1016/j.neuron.2010.08.021.

Catterall, W.A. (2012). Voltage-gated sodium channels at 60: structure, function and pathophysiology. J. Physiol. 590(11):2577-89. doi: 10.1113/jphysiol.2011.224204.

Chiara, D. C., Gill, J. F., Chen, Q., Tillman, T., Dailey, W. P., Eckenhoff, R. G., ... Cohen, J. B. (2013). Photoaffinity labeling the propofol binding site in GLIC. Biochemistry, 53(1), 135-142. doi: $10.1021 / \mathrm{bi} 401492 \mathrm{k}$.

Covarrubias, M., Barber, A. F., Carnevale, V., Treptow, W., \& Eckenhoff, R. G. (2015). Mechanistic Insights into the Modulation of Voltage-Gated Ion Channels by Inhalational Anesthetics. Biophys. J., 109(10), 2003-2011. doi: 10.1016/j.bpj.2015.09.032.

Eckenhoff, R., \& Tang, P. (2018). Recent progress on the molecular pharmacology of propofol. F1000Research, 7, 123. doi: 10.12688/f1000research.12502.1.

Essmann, U., Perera, L., Berkowitz, M. L., Darden, T., Lee, H., and Pedersen, L. G. (1995). A Smooth Particle Mesh Ewald Method. J. Chem. Phys. 103, 8577-8593.

Gamal El-Din, T.M., Lenaeus, M.J., Ramanadane, K., Zheng, N., Catterall, W.A. (2019) 
Molecular dissection of multiphase inactivation of the bacterial sodium channel NavAb. J. Gen. Physiol. 151:174-185. doi.org/10.1085/jgp.201711884.

598

Gao, S., Valinsky, W.C., On, N.C., Houlihan, P.R., Qu, Q., Liu, L., Pan, X., Clapham, D.E,. Yan, N. (2020). Employing $\mathrm{NaChBac}$ for cryo-EM analysis of toxin action on voltage-gated $\mathrm{Na}^{+}$channels in nanodisc. Proc Natl Acad Sci U S A. 117(25):14187-14193. doi: 10.1073/pnas.1922903117

Hall, M. A., Xi, J., Lor, C., Dai, S., Pearce, R., Dailey, W. P., \& Eckenhoff, R. G. (2010). m-Azipropofol (AziPm) a photoactive analogue of the intravenous general anesthetic propofol. J. Med. Chem., 53(15), 5667-75. doi: 10.1021/jm1004072.

Hemmings, H. C., Riegelhaupt, P. M., Kelz, M. B., Solt, K., Eckenhoff, R. G., Orser, B. A., \& Goldstein, P. A. (2019). Towards a Comprehensive Understanding of Anesthetic Mechanisms of Action: A Decade of Discovery. Trends Pharmacol. Sci., 40(7), 464-481. doi: 10.1016/j.tips.2019.05.001.

Herold, K. F., \& Hemmings, H. C. (2012). Sodium channels as targets for volatile anesthetics. Front. Pharmacol., 3, 50. doi: 10.3389/fphar.2012.00050.

Jayakar, S. S., Zhou, X., Chiara, D. C., Dostalova, Z., Savechenkov, P. Y., Bruzik, K. S., .. Cohen, J. B. (2014). Multiple Propofol-binding Sites in a $\gamma$-Aminobutyric Acid Type A Receptor (GABA A R) Identified Using a Photoreactive Propofol Analog. J. Biol. Chem., 289(40), 27456-27468. doi: 10.1074/jbc.M114.581728.

Jiang, D., Shi, H., Tonggu, L., Gamal El-Din, T.M., Lenaeus, M.J., Zhao, Y., Yoshioka, C., Zheng, N., Catterall, W.A. (2020). Structure of the Cardiac Sodium Channel. Cell 180(1):122-134.e10. doi:10.1016/j.cell.2019.11.041.

Jorgensen, W. L., Chandrasekhar, J., Madura, J. D., Impey, R. W. \& Klein, M. L. (1983). Comparison of Simple Potential Functions for Simulating Liquid Water. J. Chem. Phys. 79, 926-935.

Kinde, M. N., Bondarenko, V., Granata, D., Bu, W., Grasty, K. C., Loll, P. J., .. Xu, Y. (2016). Fluorine19 NMR and computational quantification of isoflurane binding to the voltage-gated sodium channel NaChBac. Proc. Natl. Acad. Sci. U. S. A., 113(48), 13762-13767. doi: 10.1073/pnas.1609939113.

Lee, S., Goodchild, S. J., \& Ahern, C. A. (2012). Local anesthetic inhibition of a bacterial sodium channel. J. Gen. Physiol., 139(6), 507-16. doi: 10.1085/jgp.201210779. 
Lingamaneni, R., Birch, M. L., \& Hemmings, H. C., Jr. (2001). Widespread inhibition of sodium channel-dependent glutamate release from isolated nerve terminals by isoflurane and propofol. Anesthesiology, 95, 1460-6 (2001).

Mackerell, A. D., Feig, M., \& Brooks, C. L. (2004). Extending the treatment of backbone energetics in protein force fields: limitations of gas-phase quantum mechanics in reproducing protein conformational distributions in molecular dynamics simulations. J. Computat Chem 25, 1400-1415.

Ouyang, W., Jih, T.-Y., Zhang, T.-T., Correa, A. M., \& Hemmings, H. C. (2007). Isoflurane inhibits NaChBac, a prokaryotic voltage-gated sodium channel. J. Pharmacol. Exp. Ther., 322(3), 1076-1083. doi: 10.1124/jpet.107.122929.lus.

Ouyang, W., Wang, G., \& Hemmings, H. C. (2003). Isoflurane and propofol inhibit voltage-gated sodium channels in isolated rat neurohypophysial nerve terminals. Mol. Pharmacol., 64(2), 373-81. doi: 10.1124/mol.64.2.373.

Pan X, Li Z, Zhou Q, Shen H, Wu K, Huang X, Chen J, Zhang J, Zhu X, Lei J, Xiong W, Gong H, Xiao B, Yan N. (2018). Structure of the human voltage-gated sodium channel $\mathrm{Na}(\mathrm{v}) 1.4$ in complex with $\beta 1$. Science 362(6412):eaau2486. doi: 10.1126/science.aau2486.

Pavlov, E., Bladen, C., Winkfein, R., Diao C., Dhaliwal, P, French, R. J. (2005). The pore, not cytoplasmic domains, underlies inactivation in a prokaryotic sodium channel. Biophys. J. 89:232-42. doi: 10.1529/biophysj.104.056994.

Phillips, J. C., Braun, R., Wang, W., Gumbart, J., Tajkhorshid, E., Villa, E., Chipot, C., Skeel, R. D., Kale, L., \& Schulten, K. (2005). Scalable molecular dynamics with NAMD. J Comput Chem 26, 1781-1802.

Ratnakumari, L., \& Hemmings, H. C., Jr. (1997). Effects of propofol on sodium channel-dependent sodium influx and glutamate release in rat cerebrocortical synaptosomes. Anesthesiology, 86, 428-39.

Sahinovic, M. M., Struys, M. M. R. F., \& Absalom, A. R. (2018, December 1). Clinical Pharmacokinetics and Pharmacodynamics of Propofol. Clin. Pharmacokinet. Springer International Publishing. doi: 10.1007/s40262-018-0672-3.

Sand, R. M., Gingrich, K. J., Macharadze, T., Herold, K. F., \& Hemmings, H. C. (2017). Isoflurane modulates activation and inactivation gating of the prokaryotic $\mathrm{Na}(+)$ channel $\mathrm{NaChBac} . J$. Gen. 
Physiol., 149(6), 623-638. doi: 10.1085/jgp.201611600.

651 Sula, A., Booker, J., Ng, L. C., Naylor, C. E., DeCaen, P. G., \& Wallace, B. A. (2017)

652 The complete structure of an activated open sodium channel. Nat. Commun. 8:14205.

653 doi: $10.1038 /$ ncomms 14205.

654 Vanommeslaeghe, K., \& MacKerell, A. D., Jr. (2012). Automation of the CHARMM General Force Field 655 (CGenFF) I: bond perception and atom typing. J. Chem. Inf. Model 52, 3144-3154.

656 Vanommeslaeghe, K., Raman, E. P., \& MacKerell, A. D., Jr. (2012). Automation of the CHARMM General 657 Force Field (CGenFF) II: assignment of bonded parameters and partial atomic charges. J. Chem. Inf. $658 \quad$ Model 52, 3155-3168.

Walsh, C. T. (2018). Propofol: milk of amnesia. Cell 175(1), 10-13. doi: 10.1016/j.cell.2018.08.031.

Wang, Y., Yang, E., Wells, M. M., Bondarenko, V., Woll, K., Carnevale, V., ... Xu, Y. (2018). Propofol inhibits the voltage-gated sodium channel NaChBac at multiple sites. J. Gen. Physiol., 150(9), 13171332. doi: 10.1085/jgp.201811993.

Waterhouse, A., Bertoni, M., Bienert, S., Studer, G., Tauriello, G., Gumienny, R., Heer, F.T., de Beer,

Weiser, B. P., Kelz, M. B., \& Eckenhoff, R. G. (2013). In vivo activation of azipropofol prolongs anesthesia

Wisedchaisri, G., Tonggu, L., McCord, E., Gamal El-Din, T. M., Wang, L., Zheng, N., \& Catterall, W. A. (2019). Resting-State Structure and Gating Mechanism of a Voltage-Gated Sodium Channel. Cell, 178(4), 993-1003.e12. doi: 10.1016/j.cell.2019.06.031.

Woll, K. A., Dailey, W. P., Brannigan, G., \& Eckenhoff, R. G. (2016). Shedding Light on Anesthetic

Woll, K. A., Skinner, K. A., Gianti, E., Bhanu, N. V., Garcia, B. A., Carnevale, V., ... Gaudet, R. (2017). Labeling. Biophys. J. 113, 2168-2172. doi: 10.1016/J.BPJ.2017.08.040. 
677 Woll, K. A., Weiser, B. P., Liang, Q., Meng, T., McKinstry-Wu, A., Pinch, B., ... Eckenhoff, R. G. (2015).

678 Role for the propofol hydroxyl in anesthetic protein target molecular recognition. ACS Chem.

$679 \quad$ Neurosci., 6(6), 927-35. doi: 10.1021/acschemneuro.5b00078.

680 Woll, K. A., Zhou, X., Bhanu, N. V., Garcia, B. A., Covarrubias, M., Miller, K. W., \& Eckenhoff, R. G. 681 (2018). Identification of binding sites contributing to volatile anesthetic effects on GABA type A 682 receptors. FASEB J., 32, 4172-4189. doi: 10.1096/fj.201701347R.

683 Yang, E., Granata, D., Eckenhoff, R. G., Carnevale, V., \& Covarrubias, M. (2018). Propofol inhibits 684 prokaryotic voltage-gated $\mathrm{Na}+$ channels by promoting activation-coupled inactivation. J. Gen. 685 Physiol., 150(9), 1299-1316. doi: 10.1085/jgp.201711924.

686 Zhou, C., Johnson, K. W., Herold, K. F., \& Hemmings, H. C. (2019). Differential inhibition of neuronal 687 sodium channel subtypes by the general anesthetic isoflurane. J. Pharmacol. Exp. Ther., 369(2), 200688 211. doi: $10.1124 /$ jpet.118.254938. 
Table 1. Changes in gating parameters of $\mathrm{NaChBac}$ induced by $5 \mu \mathrm{M}$ propofol, $\mathrm{AziPm}$ and fropofol

\begin{tabular}{|c|c|c|c|}
\hline Parameter* & Propofol $^{\S}$ & AziPm & Fropofol \\
\hline \multicolumn{4}{|c|}{ Activation NaChBac WT } \\
\hline$\Delta V_{1 / 2}(\mathrm{mV})$ & $\begin{array}{c}-9.82 \pm 1.82 * * * \\
n=15\end{array}$ & $\begin{array}{c}-12.34 \pm 1.19^{* * * *} \\
n=6\end{array}$ & $\begin{array}{c}-15.27 \pm 0.81^{* * *} \\
n=8\end{array}$ \\
\hline$\Delta z\left(\mathrm{e}_{0}\right)$ & $\begin{array}{c}-0.45 \pm 0.18^{*} \\
n=15\end{array}$ & $\begin{aligned} 0.13 & \pm 0.11 \\
n & =6\end{aligned}$ & $\begin{aligned} 0.14 & \pm 0.20 \\
n & =8\end{aligned}$ \\
\hline$\Delta G_{\max }(\%)$ & $\begin{array}{c}-2.2 \pm 4.2 \\
n=15\end{array}$ & $\begin{array}{l}8 \pm 7 \\
n=6\end{array}$ & $\begin{array}{l}-5 \pm 6 \\
n=8\end{array}$ \\
\hline$\Delta I_{\max }(\%)$ & $\begin{array}{c}11 \pm 5.1 \\
n=15\end{array}$ & $\begin{aligned} 28 & \pm 8^{*} \\
n & =6\end{aligned}$ & $\begin{array}{c}18 \pm 7^{*} \\
n=8\end{array}$ \\
\hline \multicolumn{4}{|c|}{ Activation NaChBAc T220A } \\
\hline$\Delta V_{1 / 2}(\mathrm{mV})$ & $\begin{aligned}-14.16 & \pm 0.93^{* * *} \\
n & =11\end{aligned}$ & - & $\begin{aligned}-14.72 & \pm 1.38^{* *} \\
n & =4\end{aligned}$ \\
\hline$\Delta z\left(\mathrm{e}_{0}\right)$ & $\begin{array}{c}0.01 \pm 0.25 \\
n=11\end{array}$ & - & $\begin{array}{c}-0.57 \pm 0.73 \\
n=4\end{array}$ \\
\hline$\Delta G_{\max }(\%)$ & $\begin{array}{c}0.32 \pm 7.5 \\
n=11\end{array}$ & - & $\begin{array}{c}11 \pm 9 \\
n=4\end{array}$ \\
\hline$\Delta I_{\max }(\%)$ & $\begin{aligned} 11 & \pm 8^{* *} \\
n & =11\end{aligned}$ & - & $\begin{array}{c}24 \pm 9 \\
n=4\end{array}$ \\
\hline \multicolumn{4}{|c|}{ Inactivation NaChBac WT } \\
\hline$\Delta V_{1 / 2}(\mathrm{mV})$ & $\begin{array}{c}-7.02 \pm 1.29 * * * \\
n=8\end{array}$ & $\begin{array}{c}-18.95 \pm 1.81 * * * \\
n=6\end{array}$ & $\begin{array}{c}-10.29 \pm 0.68^{* * *} \\
n=8\end{array}$ \\
\hline$\Delta z\left(\mathrm{e}_{0}\right)$ & $\begin{array}{c}0.38 \pm 0.9 \\
n=8\end{array}$ & $\begin{aligned} 0.35 & \pm 0.22 \\
n & =6\end{aligned}$ & $\begin{array}{c}-0.24 \pm 0.39 \\
n=8\end{array}$ \\
\hline $\begin{array}{l}\tau_{\text {INACTIVATION }} \\
\left(\text { fold change at } I_{\max }\right)\end{array}$ & $\begin{array}{c}-0.28 \pm 0.03 * * * \\
n=8\end{array}$ & $\begin{array}{c}-0.60 \pm 0.05^{* *} \\
n=6\end{array}$ & $\begin{array}{c}-0.22 \pm 0.06^{* *} \\
n=8\end{array}$ \\
\hline
\end{tabular}

* All values represent the mean \pm SEM of $n$ independent measurements (i.e., $n$ cells). $\Delta V_{1 / 2}$ and $\Delta z$ represent an absolute difference. The $\Delta I_{\max }$ was determined from peak current-voltage relations (Figure $\mathrm{S} 1$ ). Negative changes in $\mathrm{z}, G_{\max }, I_{\max }$, and $\tau_{\mathrm{INACTIVATION}}$ indicate decreases in these parameters. $*<0.05 ; * *<0.01$; $* * *<0.001$ (paired Student t-test to evaluate changes relative a corresponding control on the same cell in the absence of the compound)

$\S$ Values were taken from Yang et al. (Yang et al., 2018). 
Table 2. AziPm Adduction of Prokaryotic Nav Channels in Multiple Independent Assays

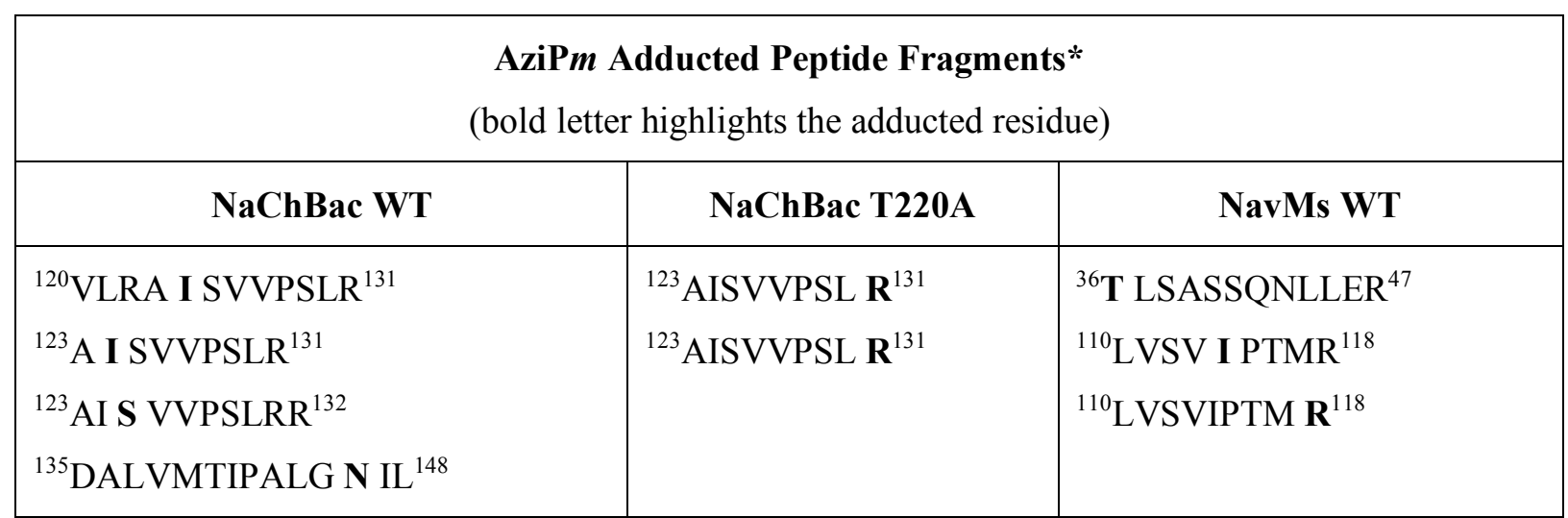

* Each peptide sequence correspond to an independent experiment. The mass spectroscopy results and \% sequence coverage from each individual attempt are shown under Supplemental Information (Figures S3a-

702 d, S4a-b, S5a-c).

703 


\section{FIGURE 1}
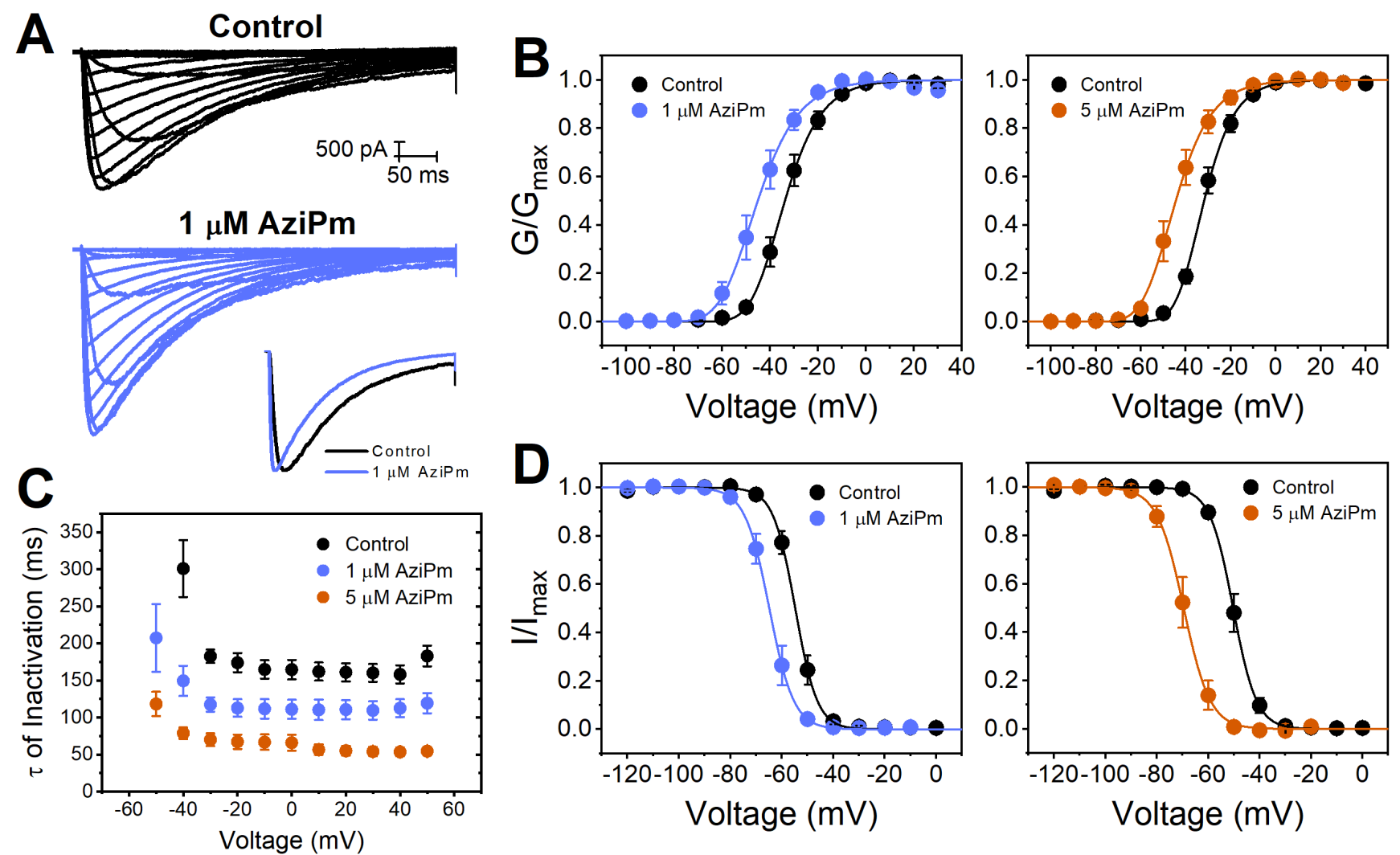

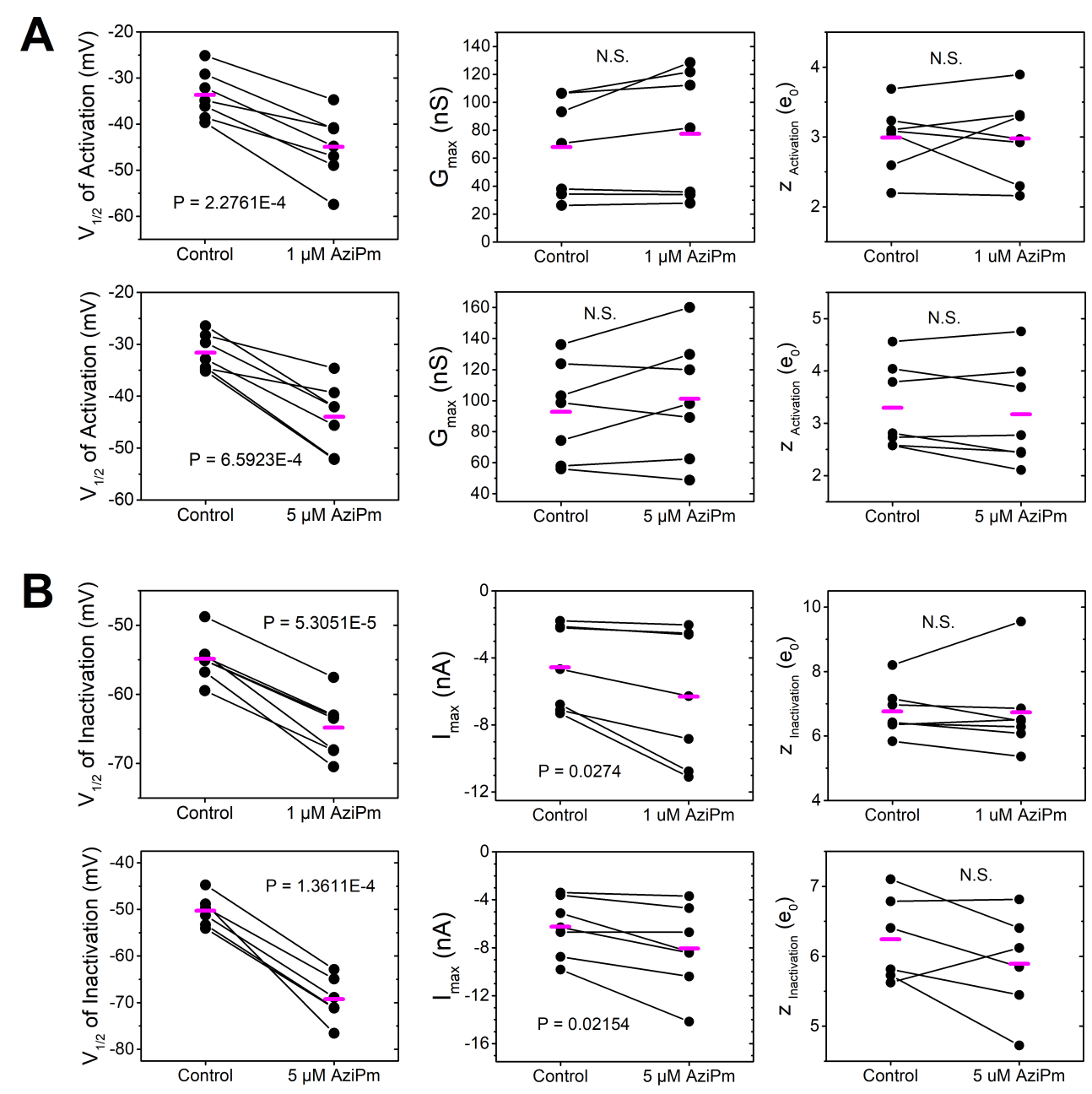

FIGURE 2 


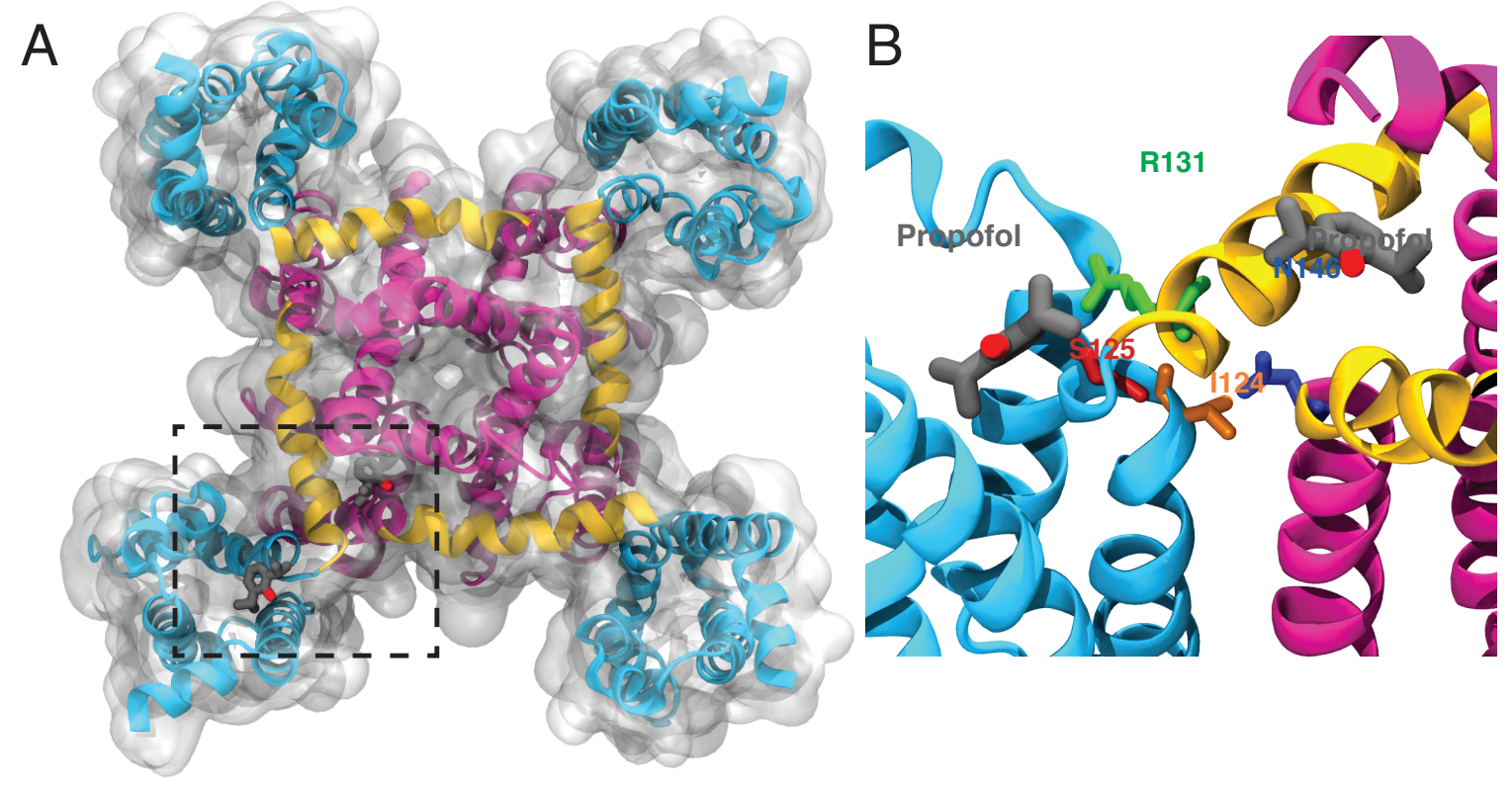

FIGURE 3

C

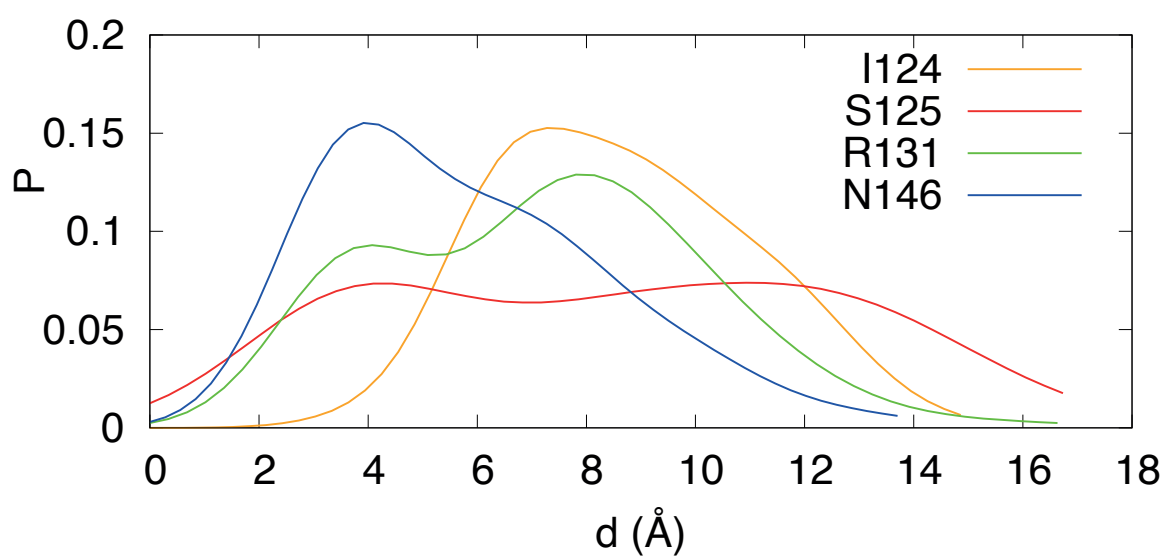


FIGURE 4

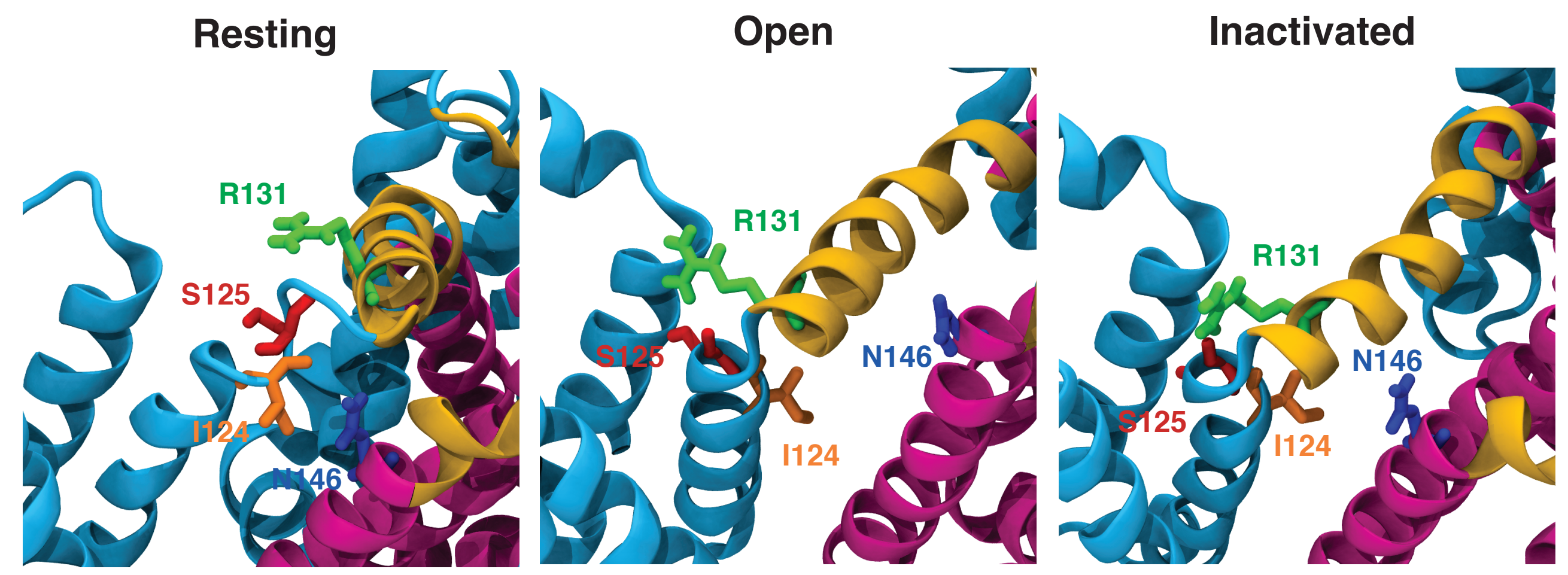



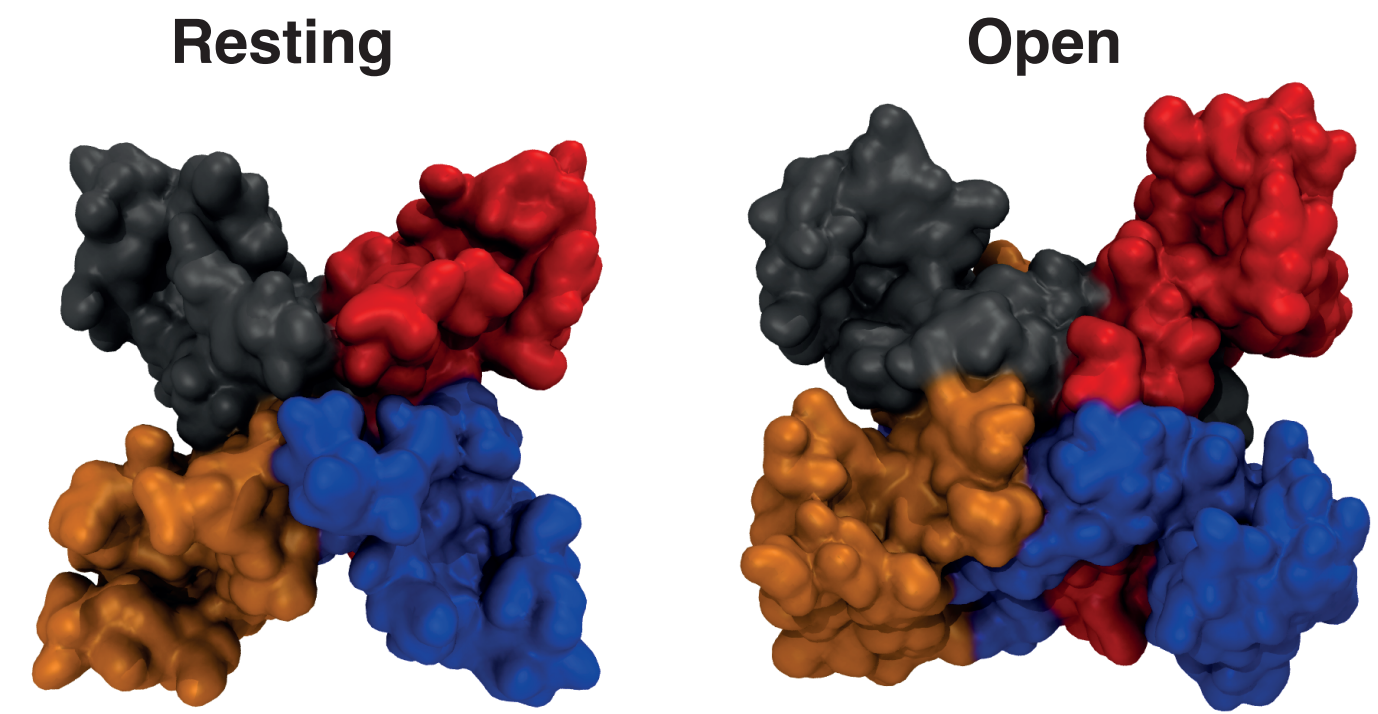

FIGURE 5

Inactivated
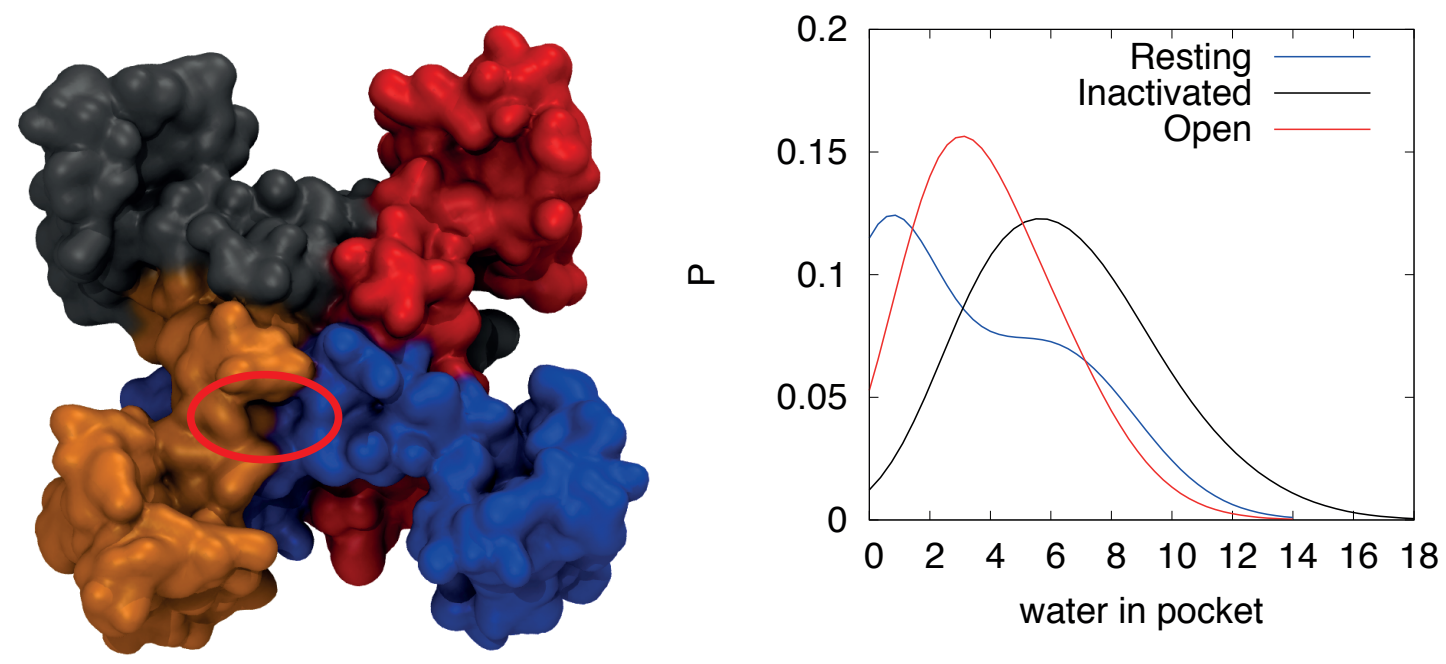


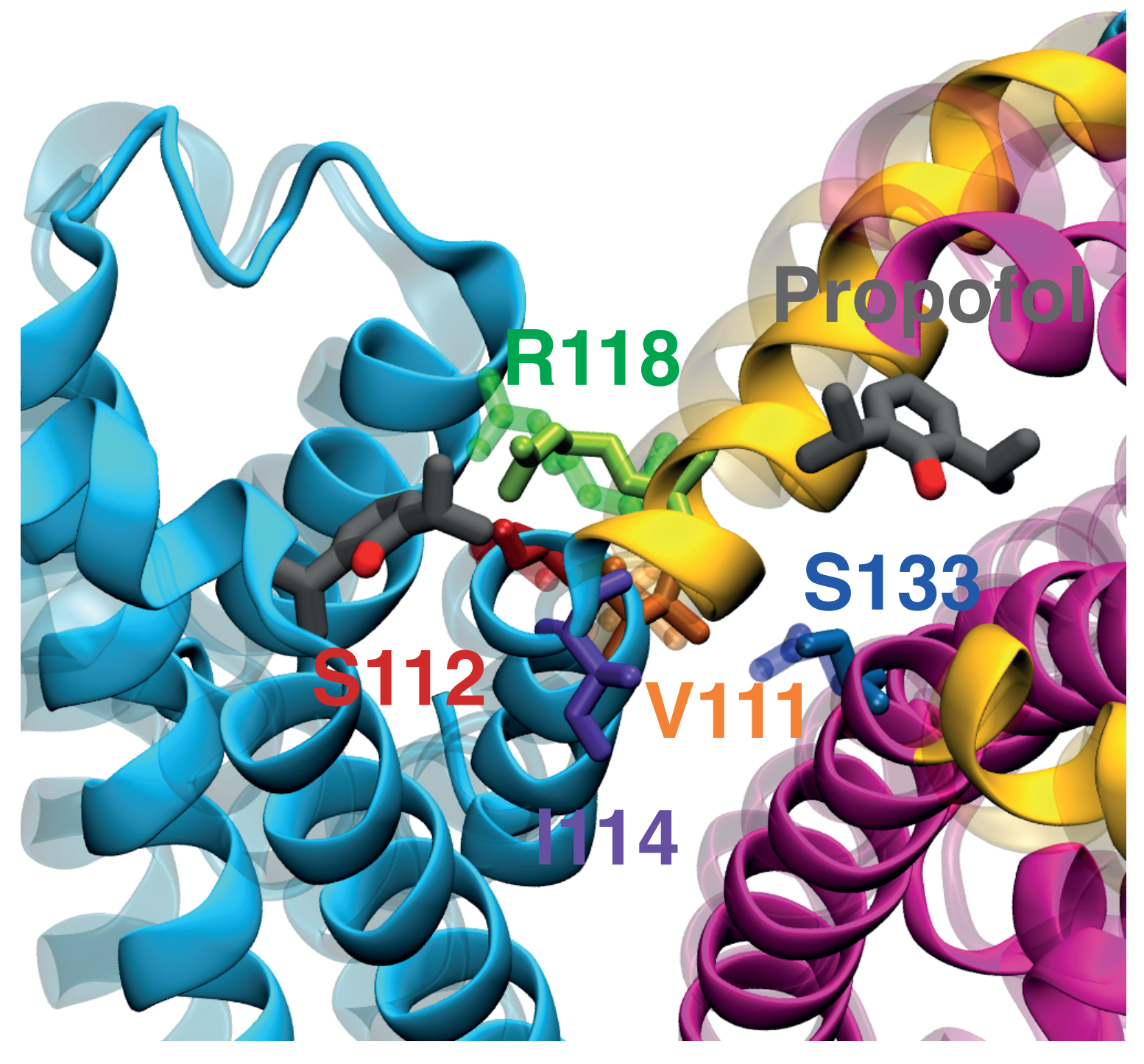

FIGURE 6 
A

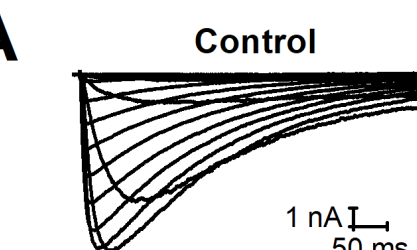

$50 \mathrm{~ms}$
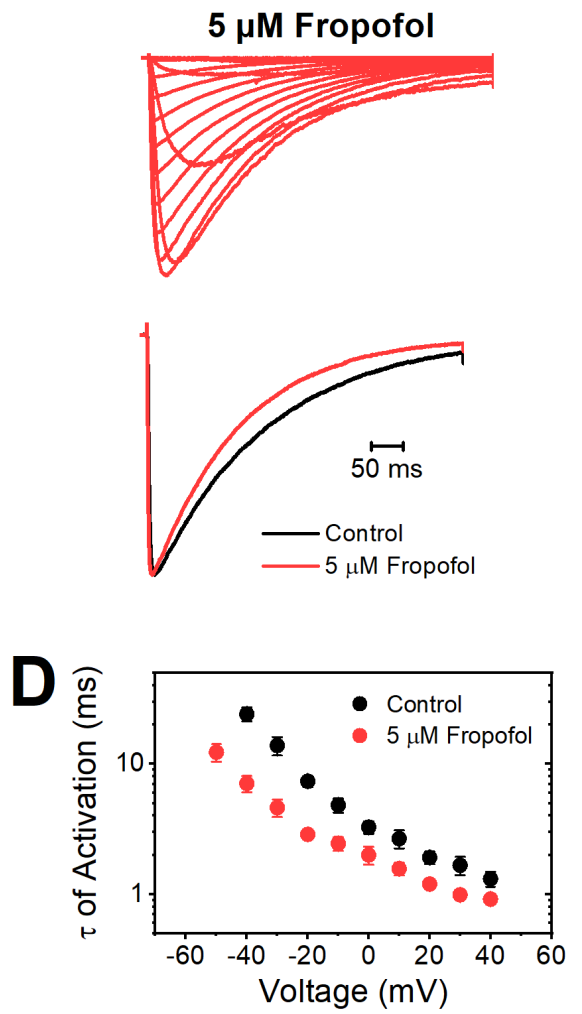
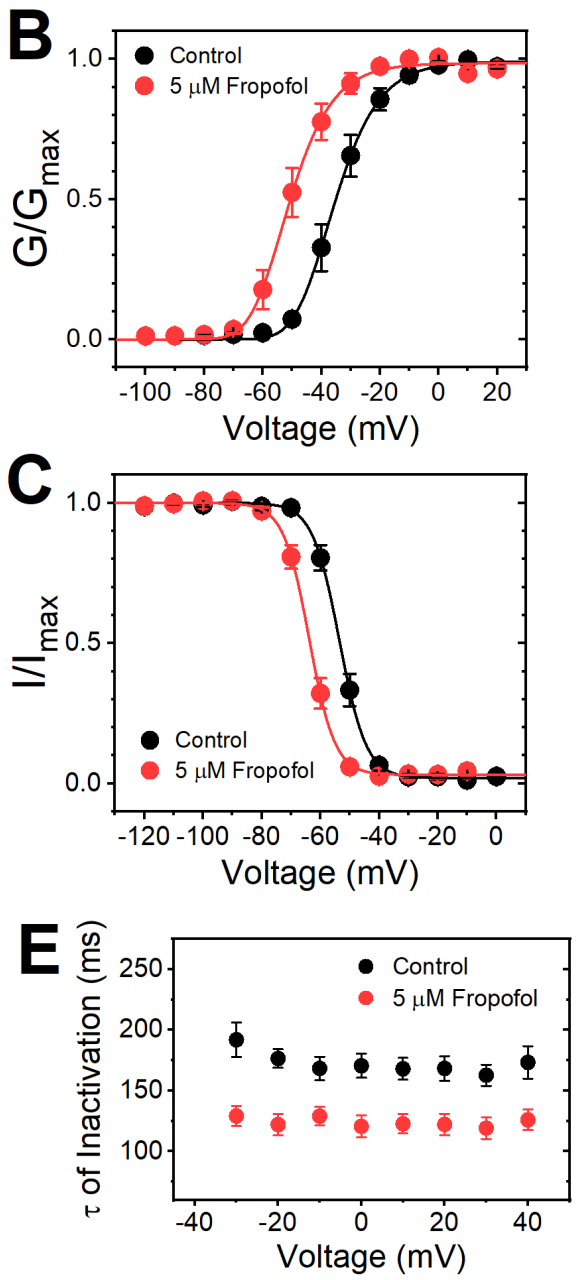

FIGURE 7 